\title{
Delay-Compensation-Based State Estimation for Time-Varying Complex Networks with Incomplete Observations and Dynamical Bias
}

\author{
Jun $\mathrm{Hu}$, Member, IEEE, Zidong Wang, Fellow, IEEE and Guo-Ping Liu, Fellow, IEEE
}

\begin{abstract}
In this paper, a delay-compensation-based state estimation (DCBSE) method is given for a class of discrete timevarying complex networks (DTVCNs) subject to network-induced incomplete observations (NIIOs) and dynamical bias. The NIIOs include the communication delays and fading observations, where the fading observations are modelled by a set of mutually independent random variables. Moreover, the possible bias is taken into account, which is depicted by a dynamical equation. A predictive scheme is proposed to compensate the influences induced by the communication delays, where the predictivebased estimation mechanism is adopted to replace the delayed estimation transmissions. This paper focuses on the problems of estimation method design and performance discussions for addressed DTVCNs with NIIOs and dynamical bias. In particular, a new distributed state estimation approach is presented, where a locally minimized upper bound is obtained for the estimation error covariance matrix and a recursive way is designed to determine the estimator gain matrix. Furthermore, the performance evaluation criteria regarding the monotonicity are proposed from the analytic perspective. Finally, some experimental comparisons are proposed to show the validity and advantages of new DCBSE approach.
\end{abstract}

Index Terms-Time-varying stochastic complex networks; Delay-compensation-based estimation; Communication delays; Incomplete Observations; Dynamical bias; Monotonicity analysis.

\section{INTRODUCTION}

Over the past years, the investigation of the complex networks (CNs) has become an emerging research topic due to its strong advantages on modelling/analyzing the practical systems, such as biological networks, power grid, traffic networks and technological networks [35], [40], [41]. Accordingly, a rich body of analysis strategies has been given to understand the structure and dynamical characteristic of $\mathrm{CNs}$ with a

This work was supported in part by the National Natural Science Foundation of China under Grants 61673141, 61933007, 61873148 and 61773144, the Outstanding Youth Science Foundation of Heilongjiang Province of China under grant JC2018001, the Fundamental Research Foundation for Universities of Heilongjiang Province of China under Grant 2019-KYYWF-0215, the European Regional Development Fund and Sêr Cymru Fellowship under Grant 80761-USW-059, and the Alexander von Humboldt Foundation of Germany. (Corresponding author: Jun Hu.)

$\mathrm{J}$. Hu is with the Department of Mathematics, Harbin University of Science and Technology, Harbin 150080, China. He is also with the School of Engineering, University of South Wales, Pontypridd CF37 1DL, United Kingdom. (Email: hujun2013@gmail.com)

Z. Wang is with the Department of Computer Science, Brunel University London, Uxbridge, Middlesex, UB8 3PH, United Kingdom. (Email: Zidong.Wang@brunel.ac.uk)

G.-P. Liu is with the Department of Artificial Intelligence and Automation, Wuhan University, Wuhan 430072, China. (Email: guoping.liuesouthwales.ac.uk) large number of coupled nodes [10], [12], [37]. Among the exiting results, both the state estimation and synchronization problems have gained the primary interest and become two important research topics for CNs [6], [27], [32]. So far, a great number of sufficient criteria combined with certain performance requirements have been proposed to handle the synchronization problems for $\mathrm{CNs}$ with different coupling impacts [7], [13], [14]. In comparison with the synchronization problem, special effort has been devoted on the state estimation problems in order to better understand the intrinsic features of the networks by properly taking the coupling relationships and shared information into account [11], [34]. Recently, the dynamics behaviour analysis of $\mathrm{CNs}$ subject to timevarying characteristics has received increasing attention on the estimation of node's state [11], [17], [18]. For example, a new estimation scheme under the error variance constraint has been presented in [11] based on the recursive matrix inequality technique, where both the changeable typology structure and quantized measurements of time-varying $\mathrm{CNs}$ have been taken into consideration and examined accordingly. In [19], the recursive estimation method under the error covariance optimization has been developed, where both the time-varying characteristics of concerned CNs and stochastic impacts of the noises have been adequately reflected in the proposed estimation strategy for the first time. Subsequently, the estimation method in [19] has been extended to propose the recursive estimation algorithms for time-varying CNs with random/nonlinear coupling influences in [28], [29].

As it is well known, the practical systems are commonly influenced by different type disturbances/inputs, which should be properly handled during the system modelling and analysis [9], [15], [46]. Consequently, special attention has been made on dealing with the joint estimation problem of unknown inputs and system states [24], [38]. For example, some optimal estimation criteria in the minimum mean square error (MMSE) sense have been established in [25], [44] for linear stochastic systems, in which both unknown inputs and system states have been well estimated within a unified framework. In [21], as a special type of unknown inputs, the stochastic bias has been modeled by a dynamical equation. Subsequently, the state estimation problems have received ongoing research attention for dynamical systems with stochastic bias [22], [23], [42]. To be more specific, the two-step estimation schemes in the MMSE sense have been given in [20] for system with unknown dynamical bias and in [23] to deal with the incomplete information situation of dynamical bias via the adaptive mech- 
anism. Very recently, the state estimation problem under the minimization consideration of the estimation error covariance has been dealt with in [42] for two-dimensional stochastic systems, where a locally optimized estimation criterion has been developed against the dynamical bias and the random parameter perturbations simultaneously. So far, the design problem of state estimation algorithm has not been discussed for discrete time-varying complex networks (DTVCNs) with dynamical bias despite its practical importance, which deserves further investigation.

In the networked communication environment, the transmissions among different nodes/components may undergo the network-enhanced complexities due to the limited capability of communication channels [8], [33], [47], [48]. As such, accompanying with the increasing popularity of networked systems, the analysis problems with respect to missing measurements and communication delays have become active research topics [4], [26], [39], [45]. For example, the state estimation problems have been tackled in [31] for time-invariant neural networks subject to multiple missing measurements and in [3] for large and diverse road networks with missing data. To cater the time-varying feature, new optimized estimation scheme of the recursive manner has been proposed in [19] for discretetime CNs subject to missing measurements. Nevertheless, it is noticed that most available state estimation algorithms against the missing measurements can applicable for handling the completely lost or successfully transferred cases only, and the estimation methods corresponding to fading observations with partial missing measurement are very few [16]. Apart from the missing/fading measurements mentioned above, the existence of the communication delays is another reason, which causes the deterioration of the estimation accuracy [1], [43]. However, it is worthwhile to point out that most of the existing state estimation methods have relied on the delay information only and the potential impacts of the communication delays among different nodes have not been taken into account [2], [5]. For the purpose of performance improvements, the predictivebased idea has been preliminarily introduced to deal with the control problems subject to communication delays [30], [36]. To the best of our knowledge, few methods are available to handle the state estimation problem for DTVCNs with fading observations and communication delays. Consequently, there is an urgent need to address the state estimation problem for DTVCNs and develop a predictive compensation estimation scheme accordingly.

Inspired by the aforementioned discussions, this paper is concerned with the delay-compensation-based state estimation (DCBSE) problem for a class of DTVCNs, where the effects from the communication delays, fading observations and dynamical bias disturbances are handled. Compared with existing state estimation problems, the major challenges of conducted DCBSE problem lie in: i) how to better characterize the network-induced incomplete observations (NIIOs) as well as dynamical bias disturbances and examine the corresponding influences on the estimation performance accordingly? ii) how to propose an efficient estimation scheme applicable for the real-time calculation? and iii) how to develop an analysis method with rigorous theoretical proof on the estimation performance evaluation? To be more specific, the NIIOs including communication delays and fading observations are taken into account within a same framework, in which a hybrid compensation method is proposed to attenuate the impacts caused by NIIOs during the estimation algorithm design. The dynamical bias disturbances are described and estimated as a by-product when estimating the node states. In addition, the algorithm evaluation criteria are provided to further reveal the engineering insight via the theoretical analysis. The major contributions can be highlighted from the following three aspects. 1) The state estimation problem in the variance-constrained sense is, for the first time, discussed for DTVCNs with NIIOs and dynamical bias. 2) Both the NIIOs and dynamical bias are well discussed, and a distributed DCBSE algorithm is developed accordingly, which possesses a recursive manner applicable for online implementations. In particular, the active compensation way based on the predictive idea is proposed and the communication delays are well examined accordingly. 3) The monotonicity analyses are provided to discuss the impacts from the fading observations described by different modelling ways onto the estimation performance. Finally, some comparative experiments are made, and both the validity and advantages of newly proposed DCBSE approach are illustrated.

The rest of the paper is arranged as follows. In Section II, both the problem formulation and preliminaries are briefly introduced, where a new delay-compensation-based estimator is designed by taking the impacts of the communication delays and fading observations into account. In Section III, the recursions of estimation error covariance matrix and its related upper bound are provided, moreover, the optimized estimator gain matrix is presented by solving a recursive matrix equation (RME). Subsequently, in Section IV, the performance evaluation problems are conducted to discuss the monotonicity analyses of the presented state estimation algorithm and the corresponding theoretical proofs are proposed accordingly. The simulations with comparative discussions are given in Section $\mathrm{V}$ to demonstrate the effectiveness of new DCBSE algorithm and some conclusions are pointed out in Section VI.

Notations. Throughout this paper, $\mathbb{R}^{n}$ stands for the $n$ dimensional Euclidean space. For a matrix $A, A^{T}, A^{-1}$ and $A>0$ denote the transpose of $A$, the inverse of $A$ and $A$ being a symmetric positive-definite matrix, respectively. $\operatorname{sym}(A)$ denotes $A+A^{T}$. ० represents the Hadamard product, where $[A \circ B]_{i j}=A_{i j} \cdot B_{i j} . \mathbb{E}\{\cdot\}$ denotes the mathematical expectation of ' $'$ '. Furthermore, we assume that the algebraic calculations are compatible when the matrix dimensions are not specified.

\section{Problem Statement and Preliminary}

In this paper, we consider the following class of DTVCNs with dynamical bias:

$$
\begin{aligned}
x_{i, k+1}= & A_{i, k} x_{i, k}+\sum_{j=1}^{N} \omega_{i j, k} \Gamma x_{j, k}+B_{i, k} b_{i, k} \\
& +D_{i, k} \theta_{i, k},
\end{aligned}
$$




$$
y_{i, k}=\Psi_{i, k} C_{i, k} x_{i, k}+\eta_{i, k}, \quad i=1,2, \cdots, N
$$

where $x_{i, k}$ refers to the $n_{x}$-dimensional state of the $i$ th node, $x_{i, 0}$ represents the initial value with mean $\bar{x}_{i, 0}$, $y_{i, k}$ denotes the $n_{y}$-dimensional measurement vector, $\Omega_{k}=$ $\left[\omega_{i j, k}\right]_{N \times N}$ is the coupling strength matrix, and $\Gamma=$ $\operatorname{diag}\left\{\gamma_{1}, \gamma_{2}, \cdots, \gamma_{n}\right\}$ is the inner coupling matrix. $\Psi_{i, k}=$ $\operatorname{diag}\left\{\psi_{i 1, k}, \psi_{i 2, k}, \cdots, \psi_{i n_{y}, k}\right\}$ depicts the phenomena of the fading observations, where $\psi_{i t, k}\left(t=1,2, \cdots, n_{y}\right)$ are the random variables over the interval $\left[a_{i t}, b_{i t}\right]$ with $0 \leq a_{i t} \leq$ $b_{i t} \leq 1$. Moreover, the mathematical means and variances of random variables $\psi_{i t, k}$ are $\bar{\psi}_{i t, k}$ and $\breve{\psi}_{i t, k}$, respectively. $\theta_{i, k}$ and $\eta_{i, k}$ represent the $n_{\theta}$-dimensional process noise and $n_{y}$-dimensional measurement noise, where $\mathbb{E}\left\{\theta_{i, k}\right\}=0$, $\mathbb{E}\left\{\eta_{i, k}\right\}=0, \mathbb{E}\left\{\theta_{i, k} \theta_{i, k}^{T}\right\}=Q_{i, k}^{\theta}$ and $\mathbb{E}\left\{\eta_{i, k} \eta_{i, k}^{T}\right\}=Q_{i, k}^{\eta}$ with $Q_{i, k}^{\eta}>0$. The dynamics model of the random bias is described as follows:

$$
b_{i, k+1}=F_{i, k} b_{i, k}+\beta_{i, k},
$$

where $b_{i, k} \in \mathbb{R}^{n_{b}}$ depicts the bias of unknown magnitude. $\beta_{i, k}$ denotes the white noise, where $\mathbb{E}\left\{\beta_{i, k}\right\}=0$ and $\mathbb{E}\left\{\beta_{i, k} \beta_{i, k}^{T}\right\}=Q_{i, k}^{\beta}$ with $Q_{i, k}^{\beta}>0 . A_{i, k}, B_{i, k}, C_{i, k}, D_{i, k}$ and $F_{i, k}$ are known matrices of proper dimensions.

The random inner coupling matrix $\Gamma$ is described by:

$$
\Gamma=\bar{\Gamma}+\Delta \bar{\Gamma},
$$

where $\bar{\Gamma}=\operatorname{diag}\left\{\bar{\gamma}_{1}, \bar{\gamma}_{2}, \cdots, \bar{\gamma}_{N}\right\}$ is the nominal inner coupling matrix, and $\Delta \bar{\Gamma}$ represents the random parameter perturbation satisfying

$$
\mathbb{E}\{\Delta \bar{\Gamma}\}=0, \quad \mathbb{E}\left\{\Delta \bar{\Gamma} \Delta \bar{\Gamma}^{T}\right\} \leq \bar{\gamma} I,
$$

in which $\bar{\gamma}$ is a known positive constant.

Remark 1: It is worth pointing out that the coupling strengthens among the state elements are different if $\gamma_{i} \neq \gamma_{j}$. Hence, the random inner-coupling strengthens characterized by (4)-(5) are introduced to present more flexibility of the CNs modelling.

Subsequently, set

$$
\begin{aligned}
\vec{x}_{i, k} & =\left[\begin{array}{cc}
x_{i, k}^{T} & b_{i, k}^{T}
\end{array}\right]^{T}, \mathcal{C}_{i, k}=\left[\begin{array}{cc}
C_{i, k} & 0
\end{array}\right], \\
\mathcal{A}_{i, k} & =\left[\begin{array}{cc}
A_{i, k} & B_{i, k} \\
0 & F_{i, k}
\end{array}\right], \quad \vec{\Gamma}=\left[\begin{array}{cc}
\bar{\Gamma} & 0 \\
0 & 0
\end{array}\right], \\
\Delta \vec{\Gamma} & =\left[\begin{array}{cc}
\Delta \bar{\Gamma} & 0 \\
0 & 0
\end{array}\right], \mathcal{D}_{i, k}=\left[\begin{array}{cc}
D_{i, k} & 0 \\
0 & I
\end{array}\right], \\
\zeta_{i, k} & =\left[\begin{array}{ll}
\theta_{i, k}^{T} & \beta_{i, k}^{T}
\end{array}\right]^{T} .
\end{aligned}
$$

Then, it follows from the above notations that the following compact form can be obtained:

$$
\begin{aligned}
\vec{x}_{i, k+1} & =\mathcal{A}_{i, k} \vec{x}_{i, k}+\sum_{j=1}^{N} \omega_{i j, k}(\vec{\Gamma}+\Delta \vec{\Gamma}) \vec{x}_{j, k}+\mathcal{D}_{i, k} \zeta_{i, k}, \\
y_{i, k} & =\Psi_{i, k} \mathcal{C}_{i, k} \vec{x}_{i, k}+\eta_{i, k} .
\end{aligned}
$$

In what follows, we assume that $\theta_{i, k}, \beta_{i, k}, \psi_{i t, k}, \eta_{i, k} x_{i, 0}$ and the element of $\Delta \Gamma$ are mutually independent in $i, t$ and $k$.

Next, the time-varying state estimator in the distributed manner is designed for each node based on the related measurements and adjacent node's estimation. During the information transmission, the communication delays between the node $j$ and the node $i$ are considered, which are denoted by $\tau_{i j}$. As such, the estimation at the delayed instant can be available only during the estimator design. For the purpose of estimation accuracy improvement, the following predictive compensation rule is employed:

$$
\begin{aligned}
\hat{x}_{j, k-\tau_{i j}+1 \mid k-\tau_{i j}} & =\mathcal{A}_{j, k-\tau_{i j}} \hat{x}_{j, k-\tau_{i j}}, \\
\hat{x}_{j, k-\tau_{i j}+2 \mid k-\tau_{i j}} & =\mathcal{A}_{j, k-\tau_{i j}+1} \hat{x}_{j, k-\tau_{i j}+1 \mid k-\tau_{i j}}, \\
& \vdots \\
\hat{x}_{j, k-1 \mid k-\tau_{i j}} & =\mathcal{A}_{j, k-2} \hat{x}_{j, k-2 \mid k-\tau_{i j}}, \\
\hat{x}_{j, k \mid k-\tau_{i j}} & =\mathcal{A}_{j, k-1} \hat{x}_{j, k-1 \mid k-\tau_{i j}} .
\end{aligned}
$$

Define $\mathcal{A}_{j, \tau_{i j}} \triangleq \prod_{t=1}^{\tau_{i j}} \mathcal{A}_{j, k-t}$. According to (9), one has $\hat{x}_{j, k \mid k-\tau_{i j}}=\mathcal{A}_{j, \tau_{i j}} \hat{x}_{j, k-\tau_{i j}}$, then the predictive estimation $\hat{x}_{j, k \mid k-\tau_{i j}}$ at the current instant is derived based on the delayed estimation $\hat{x}_{j, k-\tau_{i j}}$.

For the $i$-th node, the following state estimator under the predictive compensation mechanism is constructed:

$$
\begin{aligned}
\hat{x}_{i, k+1}= & \left(\mathcal{A}_{i, k}+\omega_{i i, k} \vec{\Gamma}\right) \hat{x}_{i, k}+\sum_{j=1, j \neq i}^{N} \omega_{i j, k} \vec{\Gamma} \hat{x}_{j, k \mid k-\tau_{i j}} \\
& +\mathcal{K}_{i, k}\left(y_{i, k}-\bar{\Psi}_{i, k} \mathcal{C}_{i, k} \hat{x}_{i, k}\right),
\end{aligned}
$$

where $\hat{x}_{i, k}$ represents the estimate of state $\vec{x}_{i, k}$ at the time step $k, \bar{\Psi}_{i, k}=\operatorname{diag}\left\{\bar{\psi}_{i 1, k}, \bar{\psi}_{i 2, k}, \cdots, \bar{\psi}_{i n_{y}, k}\right\}$ and $\bar{\psi}_{i t, k}$ is the mathematical expectation of the random variable $\psi_{i t, k}(t=$ $\left.1,2, \cdots, n_{y}\right)$, and $\mathcal{K}_{i, k}$ is the estimator parameter matrix to be determined later.

Remark 2: In order to improve the estimation accuracy, there is a need to examine the network-enhanced complexities. It should be pointed out that the hybrid compensation idea is presented to attenuate the effects from NIIOs when designing the new time-varying state estimator in (10). To be more specific, the communication delays among the different nodes are taken into account and those impacts are actively compensated in (9) by employing the delayed estimation information properly. In particular, it is worthwhile to mention that a new predictive scheme is presented based on $\hat{x}_{j, k-\tau_{i j}}$ at the time step $k-\tau_{i j}$ and the desirable estimation $\hat{x}_{j, k \mid k-\tau_{i j}}$ at the time step $k$ can be obtained iteratively. Accordingly, this predictivebased scheme is given to compensate the influences caused by the communication delays during the transmissions among the adjacent nodes. Moreover, the mathematical expectation $\bar{\Psi}_{i, k}$ is also introduced in (10) to reflect the fading observations. In this paper, such a hybrid compensation estimation manner, which contains the predictive and probability-dependent mechanisms, is developed to improve the estimation algorithm performance and the corresponding estimation accuracy comparisons under different cases of NIIOs will be provided later.

Remark 3: The new state estimator in (10) under the predictive compensation mechanism has the following features: i) the communication delays among the different transmission channels and the fading observations are addressed within same framework, where a hybrid compensation estimation manner is provided; ii) a Kalman-type estimator is constructed based 
on the predictive estimation and the locally optimal estimation scheme is expected, which performs an attractive advantage in the online implementations; and iii) the dynamical bias disturbances are discussed and estimated simultaneously.

Define $e_{i, k+1} \triangleq \vec{x}_{i, k+1}-\hat{x}_{i, k+1}$ as the estimation error and $P_{i, k+1} \triangleq \mathbb{E}\left\{e_{i, k+1} e_{i, k+1}^{T}\right\}$ as the estimation error covariance matrix. Next, we are in a position to state the major objectives of the DCBSE problem for DTVCNs with NIIOs and dynamical bias, where the following three requirements are guaranteed simultaneously.

1) Design the state estimator (10) of a recursive manner and provide a sufficient criterion to look for an upper bound covariance matrix $\mathcal{P}_{i, k+1}$ with respect to $P_{i, k+1}$.

2) Present the expression form of the estimator parameter matrix $\mathcal{K}_{i, k+1}$ in order to minimize $\mathcal{P}_{i, k+1}$.

3) Provide the theoretical proofs of the monotonicity analyses between the fading observations and estimation accuracy.

\section{Main Results}

In this section, according to the definitions, both the state covariance matrix and the estimation error covariance matrix are calculated. Subsequently, a locally minimized upper bound is given regarding the estimation error covariance and the recursive expression form of the estimator parameter matrix is provided accordingly.

To begin with, let $\tilde{\Psi}_{i, k} \triangleq \Psi_{i, k}-\bar{\Psi}_{i, k}$ with $\tilde{\Psi}_{i, k}=$ $\operatorname{diag}\left\{\tilde{\psi}_{i 1, k}, \tilde{\psi}_{i 2, k}, \cdots, \tilde{\psi}_{i n_{y}, k}\right\}$. Then, it is straight to testify that $\mathbb{E}\left\{\tilde{\psi}_{i t, k}\right\}=0\left(t=1,2, \cdots, n_{y}\right)$. Next, it follows from (7) and (10) that $e_{i, k+1}$ can be given by

$$
\begin{aligned}
e_{i, k+1}= & \left(\mathcal{A}_{i, k}+\omega_{i i, k} \vec{\Gamma}-\mathcal{K}_{i, k} \bar{\Psi}_{i, k} \mathcal{C}_{i, k}\right) e_{i, k} \\
& -\mathcal{K}_{i, k} \tilde{\Psi}_{i, k} \mathcal{C}_{i, k} \vec{x}_{i, k}+\sum_{j=1}^{N} \omega_{i j, k} \Delta \vec{\Gamma} \vec{x}_{j, k} \\
& +\sum_{j=1, j \neq i}^{N} \omega_{i j, k} \vec{\Gamma}\left(\vec{x}_{j, k}-\hat{x}_{j, k \mid k-\tau_{i j}}\right) \\
& +\mathcal{D}_{i, k} \zeta_{i, k}-\mathcal{K}_{i, k} \eta_{i, k} .
\end{aligned}
$$

Firstly, the recursion of the state covariance is computed. Secondly, the recursive expressions of estimation error covariance matrix and its upper bound are presented.

Theorem 1: Consider the DTVCNs (7) with the state estimator (10). For the positive scalars $\epsilon_{i, k}$, the state covariance matrix $\vec{X}_{i, k+1}=\mathbb{E}\left\{\vec{x}_{i, k+1} \vec{x}_{i, k+1}^{T}\right\}$ satisfies:

$$
\begin{aligned}
\vec{X}_{i, k+1} \leq & \left(1+\epsilon_{i, k}\right) \mathcal{A}_{i, k} \vec{X}_{i, k} \mathcal{A}_{i, k}^{T} \\
& +N\left(1+\epsilon_{i, k}^{-1}\right) \sum_{j=1}^{N} \omega_{i j, k}^{2} \vec{\Gamma} \vec{X}_{j, k} \vec{\Gamma}^{T} \\
& +N \sum_{j=1}^{N} \omega_{i j, k}^{2} \bar{\gamma} \lambda_{\max }\left(\vec{X}_{j, k}\right) I+\mathcal{D}_{i, k} Q_{i, k}^{\zeta} \mathcal{D}_{i, k}^{T} \\
\triangleq & \mathcal{X}_{i, k+1}
\end{aligned}
$$

where $Q_{i, k}^{\zeta}=\operatorname{diag}\left\{Q_{i, k}^{\theta}, Q_{i, k}^{\beta}\right\}$.

Proof: According to the definition of state covariance matrix, it follows that

$$
\vec{X}_{i, k+1}=\mathbb{E}\left\{\vec{x}_{i, k+1} \vec{x}_{i, k+1}^{T}\right\}
$$

$$
\begin{aligned}
= & \mathcal{A}_{i, k} \vec{X}_{i, k} \mathcal{A}_{i, k}^{T}+\operatorname{sym}\left(\mathcal{E}_{i, k}\right) \\
& +\mathbb{E}\left\{\left(\sum_{j=1}^{N} \omega_{i j, k} \vec{\Gamma} \vec{x}_{j, k}\right)\left(\sum_{j=1}^{N} \omega_{i j, k} \vec{\Gamma} \vec{x}_{j, k}\right)^{T}\right\} \\
& +\mathbb{E}\left\{\left(\sum_{j=1}^{N} \omega_{i j, k} \Delta \vec{\Gamma} \vec{x}_{j, k}\right)\left(\sum_{j=1}^{N} \omega_{i j, k} \Delta \vec{\Gamma} \vec{x}_{j, k}\right)^{T}\right\} \\
& +\sum_{r=1}^{5} \operatorname{sym}\left(\mathcal{F}_{r i, k}\right)+\mathcal{D}_{i, k} \mathbb{E}\left\{\zeta_{i, k} \zeta_{i, k}^{T}\right\} \mathcal{D}_{i, k}^{T},
\end{aligned}
$$

where

$$
\begin{aligned}
\mathcal{E}_{i, k} & =\mathcal{A}_{i, k} \mathbb{E}\left\{\vec{x}_{i, k}\left(\sum_{j=1}^{N} \omega_{i j, k} \vec{\Gamma} \vec{x}_{j, k}\right)^{T}\right\}, \\
\mathcal{F}_{1 i, k} & =\mathcal{A}_{i, k} \mathbb{E}\left\{\vec{x}_{i, k}\left(\sum_{j=1}^{N} \omega_{i j, k} \Delta \vec{\Gamma} \vec{x}_{j, k}\right)^{T}\right\}, \\
\mathcal{F}_{2 i, k} & =\mathcal{A}_{i, k} \mathbb{E}\left\{\vec{x}_{i, k} \zeta_{i, k}^{T}\right\} \mathcal{D}_{i, k}^{T}, \\
\mathcal{F}_{3 i, k} & =\mathbb{E}\left\{\left(\sum_{j=1}^{N} \omega_{i j, k} \vec{\Gamma} \vec{x}_{j, k}\right)\left(\sum_{j=1}^{N} \omega_{i j, k} \Delta \vec{\Gamma} \vec{x}_{j, k}\right)^{T}\right\}, \\
\mathcal{F}_{4 i, k} & =\mathbb{E}\left\{\left(\sum_{j=1}^{N} \omega_{i j, k} \vec{\Gamma} \vec{x}_{j, k}\right) \zeta_{i, k}^{T}\right\} \mathcal{D}_{i, k}^{T}, \\
\mathcal{F}_{5 i, k} & =\mathbb{E}\left\{\left(\sum_{j=1}^{N} \omega_{i j, k} \Delta \vec{\Gamma}_{x_{j, k}}\right) \zeta_{i, k}^{T}\right\} \mathcal{D}_{i, k}^{T} .
\end{aligned}
$$

Next, it can be observed that

$$
\begin{aligned}
\operatorname{sym}\left(\mathcal{E}_{i, k}\right) \leq & \epsilon_{i, k} \mathcal{A}_{i, k} \vec{X}_{i, k} \mathcal{A}_{i, k}^{T}+\epsilon_{i, k}^{-1} \mathbb{E}\left\{\left(\sum_{j=1}^{N} \omega_{i j, k} \vec{\Gamma} \vec{x}_{j, k}\right)\right. \\
& \left.\times\left(\sum_{j=1}^{N} \omega_{i j, k} \vec{\Gamma}_{\vec{x}_{j, k}}\right)^{T}\right\},
\end{aligned}
$$

in which $\epsilon_{i, k}>0$ are constant scalars. Moreover, we obtain

$$
\begin{aligned}
& \mathbb{E}\left\{\left(\sum_{j=1}^{N} \omega_{i j, k} \vec{\Gamma} \vec{x}_{j, k}\right)\left(\sum_{j=1}^{N} \omega_{i j, k} \vec{\Gamma} \vec{x}_{j, k}\right)^{T}\right\} \\
\leq & N \sum_{j=1}^{N} \omega_{i j, k}^{2} \vec{\Gamma} \vec{X}_{j, k} \vec{\Gamma}^{T}, \\
& \mathbb{E}\left\{\left(\sum_{j=1}^{N} \omega_{i j, k} \Delta \vec{\Gamma} \vec{x}_{j, k}\right)\left(\sum_{j=1}^{N} \omega_{i j, k} \Delta \vec{\Gamma} \vec{x}_{j, k}\right)^{T}\right\} \\
\leq & N \sum_{j=1}^{N} \omega_{i j, k}^{2} \bar{\gamma} \lambda_{\max }\left(\vec{X}_{j, k}\right) I .
\end{aligned}
$$

Furthermore, we can show that $\mathcal{F}_{r i, k}(r=1,2, \cdots, 5)$ in (14) are all zero terms. As such, it follows from (13) and (15)-(17) that the inequality in (12) can be obtained readily.

Theorem 2: Consider the DTVCNs (7) with the state estimator (10). The recursion equation of $P_{i, k+1}$ can be expressed by:

$$
\begin{aligned}
& P_{i, k+1} \\
= & \left(\mathcal{A}_{i, k}+\omega_{i i, k} \vec{\Gamma}-\mathcal{K}_{i, k} \bar{\Psi}_{i, k} \mathcal{C}_{i, k}\right) P_{i, k}\left(A_{i, k}+\omega_{i i, k} \vec{\Gamma}\right.
\end{aligned}
$$




$$
\begin{aligned}
& \left.-\mathcal{K}_{i, k} \bar{\Psi}_{i, k} \mathcal{C}_{i, k}\right)^{T}+\mathcal{K}_{i, k} \mathbb{E}\left\{\tilde{\Psi}_{i, k} \mathcal{C}_{i, k} \vec{x}_{i, k} \vec{x}_{i, k}^{T} \mathcal{C}_{i, k}^{T} \tilde{\Psi}_{i, k}^{T}\right\} \mathcal{K}_{i, k}^{T} \\
& +\mathbb{E}\left\{\left(\sum_{j=1}^{N} \omega_{i j, k} \Delta \vec{\Gamma} \vec{x}_{j, k}\right)\left(\sum_{j=1}^{N} \omega_{i j, k} \Delta \vec{\Gamma} \vec{x}_{j, k}\right)^{T}\right\} \\
& +\mathbb{E}\left\{\left[\sum_{j=1, j \neq i}^{N} \omega_{i j, k} \vec{\Gamma}\left(\vec{x}_{j, k}-\hat{x}_{j, k \mid k-\tau_{i j}}\right)\right]\right. \\
& \left.\times\left[\sum_{j=1, j \neq i}^{N} \omega_{i j, k} \vec{\Gamma}\left(\vec{x}_{j, k}-\hat{x}_{j, k \mid k-\tau_{i j}}\right)\right]^{T}\right\} \\
& +\operatorname{sym}\left(\mathcal{H}_{i, k}\right)+\mathcal{D}_{i, k} Q_{i, k}^{\zeta} \mathcal{D}_{i, k}^{T}+\mathcal{K}_{i, k} Q_{i, k}^{\eta} \mathcal{K}_{i, k}^{T},
\end{aligned}
$$

where

$$
\begin{aligned}
\mathcal{H}_{i, k}= & \mathbb{E}\left\{\left(\mathcal{A}_{i, k}+\omega_{i i, k} \vec{\Gamma}-\mathcal{K}_{i, k} \bar{\Psi}_{i, k} \mathcal{C}_{i, k}\right) e_{i, k}\right. \\
& \left.\times\left[\sum_{j=1, j \neq i}^{N} \omega_{i j, k} \vec{\Gamma}\left(\vec{x}_{j, k}-\hat{x}_{j, k \mid k-\tau_{i j}}\right)\right]^{T}\right\} .
\end{aligned}
$$

Proof: Based on the definition of $P_{i, k+1}$, we have

$$
\begin{aligned}
& P_{i, k+1} \\
= & \left(\mathcal{A}_{i, k}+\omega_{i i, k} \vec{\Gamma}-\mathcal{K}_{i, k} \bar{\Psi}_{i, k} \mathcal{C}_{i, k}\right) P_{i, k}\left(A_{i, k}+\omega_{i i, k} \vec{\Gamma}\right. \\
& \left.-\mathcal{K}_{i, k} \bar{\Psi}_{i, k} \mathcal{C}_{i, k}\right)^{T}+\mathcal{K}_{i, k} \mathbb{E}\left\{\tilde{\Psi}_{i, k} \mathcal{C}_{i, k} \vec{x}_{i, k} \vec{x}_{i, k}^{T} \mathcal{C}_{i, k}^{T} \tilde{\Psi}_{i, k}^{T}\right\} \mathcal{K}_{i, k}^{T} \\
& +\mathbb{E}\left\{\left(\sum_{j=1}^{N} \omega_{i j, k} \Delta \vec{\Gamma} \vec{x}_{j, k}\right)\left(\sum_{j=1}^{N} \omega_{i j, k} \Delta \vec{\Gamma}_{\vec{x}_{j, k}}\right)^{T}\right\} \\
& +\mathbb{E}\left\{\left[\sum_{j=1, j \neq i}^{N} \omega_{i j, k} \vec{\Gamma}\left(\vec{x}_{j, k}-\hat{x}_{j, k \mid k-\tau_{i j}}\right)\right]\right. \\
& \left.+\left[\sum_{j=1, j \neq i}^{N} \omega_{i j, k} \vec{\Gamma}\left(\vec{x}_{j, k}-\hat{x}_{j, k \mid k-\tau_{i j}}\right)\right]^{T}\right\} \\
& +\mathcal{D}_{i, k} Q_{i, k}^{\zeta} \mathcal{D}_{i, k}^{T}+\mathcal{K}_{i, k} Q_{i, k}^{\eta} \mathcal{K}_{i, k}^{T}+\operatorname{sym}\left(\mathcal{H}_{i, k}\right) \\
& -\operatorname{sym}\left(\mathcal{G}_{1 i, k}\right)+\operatorname{sym}\left(\mathcal{G}_{2 i, k}\right)+\operatorname{sym}\left(\mathcal{G}_{3 i, k}\right)-\operatorname{sym}\left(\mathcal{G}_{4 i, k}\right) \\
& -\operatorname{sym}\left(\mathcal{G}_{5 i, k}\right)-\operatorname{sym}\left(\mathcal{G}_{6 i, k}\right)-\operatorname{sym}\left(\mathcal{G}_{7 i, k}\right)+\operatorname{sym}\left(\mathcal{G}_{8 i, k}\right) \\
& +\operatorname{sym}\left(\mathcal{G}_{9 i, k}\right)+\operatorname{sym}\left(\mathcal{G}_{10 i, k}\right)-\operatorname{sym}\left(\mathcal{G}_{11 i, k}\right) \\
& +\operatorname{sym}\left(\mathcal{G}_{12 i, k}\right)-\operatorname{sym}\left(\mathcal{G}_{13 i, k}\right)-\operatorname{sym}\left(\mathcal{G}_{14 i, k}\right),
\end{aligned}
$$

where

$$
\begin{aligned}
\mathcal{G}_{1 i, k}= & \mathbb{E}\left\{\left(\mathcal{A}_{i, k}+\omega_{i i, k} \vec{\Gamma}-\mathcal{K}_{i, k} \bar{\Psi}_{i, k} \mathcal{C}_{i, k}\right) e_{i, k}\right. \\
& \left.\times \vec{x}_{i, k}^{T} \mathcal{C}_{i, k}^{T} \tilde{\Psi}_{i, k}^{T} \mathcal{K}_{i, k}^{T}\right\}, \\
\mathcal{G}_{2 i, k}= & \mathbb{E}\left\{\left(\mathcal{A}_{i, k}+\omega_{i i, k} \vec{\Gamma}-\mathcal{K}_{i, k} \bar{\Psi}_{i, k} \mathcal{C}_{i, k}\right) e_{i, k}\right. \\
& \left.\times\left(\sum_{j=1}^{N} \omega_{i j, k} \Delta \vec{\Gamma} \vec{x}_{j, k}\right)^{T}\right\}, \\
\mathcal{G}_{3 i, k}= & \mathbb{E}\left\{\left(\mathcal{A}_{i, k}+\omega_{i i, k} \vec{\Gamma}-\mathcal{K}_{i, k} \bar{\Psi}_{i, k} \mathcal{C}_{i, k}\right) e_{i, k} \zeta_{i, k}^{T} \mathcal{D}_{i, k}^{T}\right\}, \\
\mathcal{G}_{4 i, k}= & \mathbb{E}\left\{\left(\mathcal{A}_{i, k}+\omega_{i i, k} \vec{\Gamma}-\mathcal{K}_{i, k} \bar{\Psi}_{i, k} \mathcal{C}_{i, k}\right) e_{i, k} \eta_{i, k}^{T} \mathcal{K}_{i, k}^{T}\right\}, \\
\mathcal{G}_{5 i, k}= & \mathbb{E}\left\{\mathcal{K}_{i, k} \tilde{\Psi}_{i, k} \mathcal{C}_{i, k} \vec{x}_{i, k}\left(\sum_{j=1}^{N} \omega_{i j, k} \Delta \vec{\Gamma} \vec{x}_{j, k}\right)^{T}\right\}, \\
\mathcal{G}_{6 i, k}= & \mathbb{E}\left\{\mathcal { K } _ { i , k } \tilde { \Psi } _ { i , k } \mathcal { C } _ { i , k } \vec { x } _ { i , k } \left[\sum_{j=1, j \neq i}^{N} \omega_{i j, k} \vec{\Gamma}\right.\right.
\end{aligned}
$$

$$
\begin{aligned}
& \left.\left.\times\left(\vec{x}_{j, k}-\hat{x}_{j, k \mid k-\tau_{i j}}\right)\right]^{T}\right\}, \\
& \mathcal{G}_{7 i, k}=\mathbb{E}\left\{\mathcal{K}_{i, k} \tilde{\Psi}_{i, k} \mathcal{C}_{i, k} \vec{x}_{i, k} \zeta_{i, k}^{T} \mathcal{D}_{i, k}^{T}\right\}, \\
& \mathcal{G}_{8 i, k}=\mathbb{E}\left\{\mathcal{K}_{i, k} \tilde{\Psi}_{i, k} \mathcal{C}_{i, k} \vec{x}_{i, k} \eta_{i, k}^{T} \mathcal{K}_{i, k}^{T}\right\}, \\
& \mathcal{G}_{9 i, k}=\mathbb{E}\left\{( \sum _ { j = 1 } ^ { N } \omega _ { i j , k } \Delta \vec { \Gamma } \vec { x } _ { j , k } ) \left[\sum_{j=1, j \neq i}^{N} \omega_{i j, k} \vec{\Gamma}\right.\right. \\
& \left.\left.\times\left(\vec{x}_{j, k}-\hat{x}_{j, k \mid k-\tau_{i j}}\right)\right]^{T}\right\} \text {, } \\
& \mathcal{G}_{10 i, k}=\mathbb{E}\left\{\left(\sum_{j=1}^{N} \omega_{i j, k} \Delta \vec{\Gamma} \vec{x}_{j, k}\right) \zeta_{i, k}^{T} \mathcal{D}_{i, k}^{T}\right\}, \\
& \mathcal{G}_{11 i, k}=\mathbb{E}\left\{\left(\sum_{j=1}^{N} \omega_{i j, k} \Delta \vec{\Gamma} \vec{x}_{j, k}\right) \eta_{i, k}^{T} \mathcal{K}_{i, k}^{T}\right\}, \\
& \mathcal{G}_{12 i, k}=\mathbb{E}\left\{\left[\sum_{j=1, j \neq i}^{N} \omega_{i j, k} \vec{\Gamma}\left(\vec{x}_{j, k}-\hat{x}_{j, k \mid k-\tau_{i j}}\right)\right] \zeta_{i, k}^{T} \mathcal{D}_{i, k}^{T}\right\}, \\
& \mathcal{G}_{13 i, k}=\mathbb{E}\left\{\left[\sum_{j=1, j \neq i}^{N} \omega_{i j, k} \vec{\Gamma}\left(\vec{x}_{j, k}-\hat{x}_{j, k \mid k-\tau_{i j}}\right)\right] \eta_{i, k}^{T} \mathcal{K}_{i, k}^{T}\right\}, \\
& \mathcal{G}_{14 i, k}=\mathcal{D}_{i, k} \mathbb{E}\left\{\zeta_{i, k} \eta_{i, k}^{T}\right\} \mathcal{K}_{i, k}^{T},
\end{aligned}
$$

and $\mathcal{H}_{i, k}$ is denoted in (19). Subsequently, it is easy to derive the recursion in (18).

Now, we are in a position to look for an upper bound $\mathcal{Q}_{i, k+1}$ of $Q_{i, k+1}$. Accordingly, the locally minimized upper bound is obtained by resorting to the mathematical induction method and completing square technique, moreover, the estimator gain matrix $\mathcal{K}_{i, k}$ is parameterized via the solution to an RME.

Theorem 3: Consider the recursion of estimation error covariance matrix in (18) and let $\mu_{i, k}>0$ be constant scalars. Under the initial condition $\mathcal{T}_{i, 0}=\vec{X}_{i, 0}>0$, if the following RME

$$
\begin{aligned}
\mathcal{T}_{i, k+1}= & \left(1+\mu_{i, k}\right)\left(\mathcal{A}_{i, k}+\omega_{i i, k} \vec{\Gamma}-\mathcal{K}_{i, k} \bar{\Psi}_{i, k} \mathcal{C}_{i, k}\right) \mathcal{T}_{i, k} \\
& \times\left(\mathcal{A}_{i, k}+\omega_{i i, k} \vec{\Gamma}-\mathcal{K}_{i, k} \bar{\Psi}_{i, k} \mathcal{C}_{i, k}\right)^{T} \\
& +2\left(1+\mu_{i, k}^{-1}\right)(N-1) \sum_{j=1, j \neq i}^{N} \omega_{i j, k}^{2} \\
& \times \vec{\Gamma}\left(\mathcal{X}_{j, k}+\hat{x}_{j, k \mid k-\tau_{i j}} \hat{x}_{j, k \mid k-\tau_{i j}}^{T}\right) \vec{\Gamma}^{T} \\
& +\mathcal{K}_{i, k}\left[\breve{\Psi}_{i, k} \circ\left(\mathcal{C}_{i, k} \mathcal{X}_{i, k} \mathcal{C}_{i, k}^{T}\right)\right] \mathcal{K}_{i, k}^{T} \\
& +N \bar{\gamma} \sum_{j=1}^{N} \omega_{i j, k}^{2} \lambda_{\max }\left(\mathcal{X}_{j, k}\right) I \\
& +\mathcal{D}_{i, k} \mathcal{Q}_{i, k}^{\zeta} \mathcal{D}_{i, k}^{T}+\mathcal{K}_{i, k} \mathcal{Q}_{i, k}^{\eta} \mathcal{K}_{i, k}^{T}
\end{aligned}
$$

has a solution $\mathcal{T}_{i, k+1}>0$, then we have

$$
P_{i, k+1} \leq \mathcal{T}_{i, k+1} .
$$

Furthermore, it is shown that $\mathcal{T}_{i, k+1}$ can be minimized if $\mathcal{K}_{i, k}$ is designed by

$$
\mathcal{K}_{i, k}=\left[\left(1+\mu_{i, k}\right)\left(\mathcal{A}_{i, k}+\omega_{i i, k} \vec{\Gamma}\right) \mathcal{T}_{i, k} \mathcal{C}_{i, k}^{T} \bar{\Psi}_{i, k}^{T}\right] \Xi_{i, k}^{-1}
$$

with

$$
\Xi_{i, k}=\left(1+\mu_{i, k}\right) \bar{\Psi}_{i, k} \mathcal{C}_{i, k} \mathcal{T}_{i, k} \mathcal{C}_{i, k}^{T} \bar{\Psi}_{i, k}^{T}+\mathcal{Q}_{i, k}^{\eta}
$$




$$
\begin{aligned}
& +\breve{\Psi}_{i, k} \circ\left(\mathcal{C}_{i, k} \mathcal{X}_{i, k} \mathcal{C}_{i, k}^{T}\right), \\
\breve{\Psi}_{i, k}= & \operatorname{diag}\left\{\breve{\psi}_{i 1, k}, \breve{\psi}_{i 2, k}, \cdots, \breve{\psi}_{i n_{y}, k}\right\} .
\end{aligned}
$$

Proof: The proof of this theorem includes two steps. That is, an upper bound for the estimation error covariance is firstly derived and a locally minimized upper bound is given in terms of the completing square technique. In addition, the expression form of the estimator parameter matrix is characterized based on the solution to an RME.

Based on the property of the Hadamard product, the second term in (18) equals to

$$
\begin{aligned}
& \mathcal{K}_{i, k} \mathbb{E}\left\{\tilde{\Psi}_{i, k} \mathcal{C}_{i, k} \vec{x}_{i, k} \vec{x}_{i, k}^{T} \mathcal{C}_{i, k}^{T} \tilde{\Psi}_{i, k}^{T}\right\} \mathcal{K}_{i, k}^{T} \\
= & \mathcal{K}_{i, k}\left[\breve{\Psi}_{i, k} \circ\left(\mathcal{C}_{i, k} \vec{X}_{i, k} \mathcal{C}_{i, k}^{T}\right)\right] \mathcal{K}_{i, k}^{T},
\end{aligned}
$$

where $\breve{\Psi}_{i, k}=\left[\mathbb{E}\left\{\tilde{\psi}_{i s, k} \tilde{\psi}_{i t, k}\right\}\right] n_{y} \times n_{y}$ and $\circ$ is the Hadamard product. Notice that $\psi_{i t, k}\left(t=1,2, \cdots, n_{y}\right)$ are mutually independent in different $s$ and $t$. Therefore, we have $\breve{\Psi}_{i, k}=\operatorname{diag}\left\{\breve{\psi}_{i 1, k}, \breve{\psi}_{i 2, k}, \cdots, \breve{\psi}_{i n_{y}, k}\right\}$.

Together with (5), one has

$$
\begin{aligned}
& \mathbb{E}\left\{\left(\sum_{j=1}^{N} \omega_{i j, k} \Delta \vec{\Gamma} \vec{x}_{j, k}\right)\left(\sum_{j=1}^{N} \omega_{i j, k} \Delta \vec{\Gamma} \vec{x}_{j, k}\right)^{T}\right\} \\
\leq & N \mathbb{E}\left\{\sum_{j=1}^{N} \omega_{i j, k}^{2} \Delta \vec{\Gamma} \vec{x}_{j, k} \vec{x}_{j, k}^{T} \Delta \vec{\Gamma}^{T}\right\} \\
\leq & N \bar{\gamma} \sum_{j=1}^{N} \omega_{i j, k}^{2} \lambda_{\max }\left(\vec{X}_{j, k}\right) I .
\end{aligned}
$$

Moreover, the fifth term in (18) is tackled as follows:

$$
\begin{aligned}
& \operatorname{sym}\left(\mathcal{H}_{i, k}\right) \\
\leq & \mu_{i, k}\left(A_{i, k}+\omega_{i i, k} \vec{\Gamma}-\bar{\lambda}_{i, k} \mathcal{K}_{i, k} C_{i, k}\right) P_{i, k} \\
& \times\left(A_{i, k}+\omega_{i i, k} \vec{\Gamma}-\bar{\lambda}_{i, k} \mathcal{K}_{i, k} C_{i, k}\right)^{T} \\
& +\mu_{i, k}^{-1} \mathbb{E}\left\{\left[\sum_{j=1, j \neq i}^{N} \omega_{i j, k} \vec{\Gamma}\left(\vec{x}_{j, k}-\hat{x}_{j, k \mid k-d_{i j}}\right)\right]\right. \\
& \left.\times\left[\sum_{j=1, j \neq i}^{N} \omega_{i j, k} \vec{\Gamma}\left(\vec{x}_{j, k}-\hat{x}_{j, k \mid k-d_{i j}}\right)\right]^{T}\right\}
\end{aligned}
$$

with $\mu_{i, k}>0$ being constant scalars. By taking (18) and (25)(27) into account, we have the following inequality

$$
\begin{aligned}
& P_{i, k+1} \\
\leq & \left(1+\mu_{i, k}\right)\left(\mathcal{A}_{i, k}+\omega_{i i, k} \vec{\Gamma}-\mathcal{K}_{i, k} \bar{\Psi}_{i, k} \mathcal{C}_{i, k}\right) P_{i, k} \\
& \times\left(A_{i, k}+\omega_{i i, k} \vec{\Gamma}-\mathcal{K}_{i, k} \bar{\Psi}_{i, k} \mathcal{C}_{i, k}\right)^{T} \\
& +\mathcal{K}_{i, k}\left[\breve{\Psi}_{i, k} \circ\left(\mathcal{C}_{i, k} \vec{X}_{i, k} \mathcal{C}_{i, k}^{T}\right)\right] \mathcal{K}_{i, k}^{T} \\
& +N \bar{\gamma} \sum_{j=1}^{N} \omega_{i j, k}^{2} \lambda_{\max }\left(\vec{X}_{j, k}\right) I \\
& +\left(1+\mu_{i, k}^{-1}\right) \mathbb{E}\left\{\left[\sum_{j=1, j \neq i}^{N} \omega_{i j, k} \vec{\Gamma}\left(\vec{x}_{j, k}-\hat{x}_{j, k \mid k-\tau_{i j}}\right)\right]\right. \\
& \left.\times\left[\sum_{j=1, j \neq i}^{N} \omega_{i j, k} \vec{\Gamma}\left(\vec{x}_{j, k}-\hat{x}_{j, k \mid k-\tau_{i j}}\right)\right]^{T}\right\} \\
& +\mathcal{D}_{i, k} Q_{i, k}^{\zeta} \mathcal{D}_{i, k}^{T}+\mathcal{K}_{i, k} Q_{i, k}^{\eta} \mathcal{K}_{i, k}^{T} .
\end{aligned}
$$

Subsequently, the following inequality can be readily obtained:

$$
\begin{aligned}
& \mathbb{E}\left\{\left[\sum_{j=1, j \neq i}^{N} \omega_{i j, k} \vec{\Gamma}\left(\vec{x}_{j, k}-\hat{x}_{j, k \mid k-\tau_{i j}}\right)\right]\right. \\
& \left.\times\left[\sum_{j=1, j \neq i}^{N} \omega_{i j, k} \vec{\Gamma}\left(\vec{x}_{j, k}-\hat{x}_{j, k \mid k-\tau_{i j}}\right)\right]^{T}\right\} \\
\leq & 2(N-1) \mathbb{E}\left\{\sum _ { j = 1 , j \neq i } ^ { N } \omega _ { i j , k } ^ { 2 } \vec { \Gamma } \left(\vec{x}_{j, k} \vec{x}_{j, k}^{T}\right.\right. \\
& \left.+\hat{x}_{j, k \mid k-\tau_{i j}} \hat{x}_{j, k \mid k-\tau_{i j}}^{T} \vec{\Gamma}^{T}\right\} \\
= & 2(N-1) \sum_{j=1, j \neq i}^{N} \omega_{i j, k}^{2} \vec{\Gamma}\left(X_{j, k}+\hat{x}_{j, k \mid k-\tau_{i j}} \hat{x}_{j, k \mid k-\tau_{i j}}^{T}\right) \vec{\Gamma}^{T} .
\end{aligned}
$$

Together with (28) and (29) leads to

$$
\begin{aligned}
& P_{i, k+1} \\
\leq & \left(1+\mu_{i, k}\right)\left(\mathcal{A}_{i, k}+\omega_{i i, k} \vec{\Gamma}-\mathcal{K}_{i, k} \bar{\Psi}_{i, k} \mathcal{C}_{i, k}\right) P_{i, k} \\
& \times\left(A_{i, k}+\omega_{i i, k} \vec{\Gamma}-\mathcal{K}_{i, k} \bar{\Psi}_{i, k} \mathcal{C}_{i, k}\right)^{T} \\
& +\mathcal{K}_{i, k}\left[\breve{\Psi}_{i, k} \circ\left(\mathcal{C}_{i, k} \vec{X}_{i, k} \mathcal{C}_{i, k}^{T}\right)\right] \mathcal{K}_{i, k}^{T} \\
& +N \bar{\gamma} \sum_{j=1}^{N} \omega_{i j, k}^{2} \lambda_{\max }\left(\vec{X}_{j, k}\right) I+2\left(1+\mu_{i, k}^{-1}\right)(N-1) \\
& \times \sum_{j=1, j \neq i}^{N} \omega_{i j, k}^{2} \vec{\Gamma}\left(X_{j, k}+\hat{x}_{j, k \mid k-\tau_{i j}} \hat{x}_{j, k \mid k-\tau_{i j}}^{T}\right) \\
& +\mathcal{D}_{i, k} Q_{i, k}^{\zeta} \mathcal{D}_{i, k}^{T}+\mathcal{K}_{i, k} Q_{i, k}^{\eta} \mathcal{K}_{i, k}^{T} .
\end{aligned}
$$

After the straightforward calculations, we arrive at

$$
P_{i, k+1} \leq \mathcal{T}_{i, k+1},
$$

where $\mathcal{T}_{i, k+1}$ is denoted in (21).

Finally, $\mathcal{T}_{i, k+1}$ in (21) can be reorganized as:

$$
\begin{aligned}
& \mathcal{T}_{i, k+1} \\
= & \left(1+\mu_{i, k}\right)\left(\mathcal{A}_{i, k}+\omega_{i i, k} \vec{\Gamma}\right) \mathcal{T}_{i, k}\left(\mathcal{A}_{i, k}+\omega_{i i, k} \vec{\Gamma}\right)^{T} \\
& +2\left(1+\mu_{i, k}^{-1}\right)(N-1) \sum_{j=1, j \neq i}^{N} \omega_{i j, k}^{2} \\
& \times \vec{\Gamma}\left(\mathcal{X}_{j, k}+\hat{x}_{j, k \mid k-\tau_{i j}} \hat{x}_{j, k \mid k-\tau_{i j}}^{T}\right) \vec{\Gamma}^{T} \\
& +N \bar{\gamma} \sum_{j=1}^{N} \omega_{i j, k}^{2} \lambda_{\max }\left(\mathcal{X}_{j, k}\right) I+\mathcal{D}_{i, k} \mathcal{Q}_{i, k}^{\zeta} \mathcal{D}_{i, k}^{T} \\
& -\left(1+\mu_{i, k}\right)\left(\mathcal{A}_{i, k}+\omega_{i i, k} \vec{\Gamma}\right) \mathcal{T}_{i, k} \mathcal{C}_{i, k}^{T} \bar{\Psi}_{i, k}^{T} \mathcal{K}_{i, k}^{T} \\
& -\left(1+\mu_{i, k}\right) \mathcal{K}_{i, k} \bar{\Psi}_{i, k} \mathcal{C}_{i, k} \mathcal{T}_{i, k}\left(\mathcal{A}_{i, k}+\omega_{i i, k} \vec{\Gamma}\right)^{T} \\
& +\mathcal{K}_{i, k}\left[\left(1+\mu_{i, k}\right) \bar{\Psi}_{i, k} \mathcal{C}_{i, k} \mathcal{T}_{i, k} \mathcal{C}_{i, k}^{T} \bar{\Psi}_{i, k}^{T}\right. \\
& \left.+\breve{\Psi}_{i, k} \circ\left(\mathcal{C}_{i, k} \mathcal{X}_{i, k} \mathcal{C}_{i, k}^{T}\right)+\mathcal{Q}_{i, k}^{\eta}\right] \mathcal{K}_{i, k}^{T} \\
= & \left(1+\mu_{i, k}\right)\left(\mathcal{A}_{i, k}+\omega_{i i, k} \vec{\Gamma}\right) \mathcal{T}_{i, k}\left(\mathcal{A}_{i, k}+\omega_{i i, k} \vec{\Gamma}\right)^{T} \\
& +2\left(1+\mu_{i, k}^{-1}\right)(N-1) \sum_{j=1, j \neq i}^{N} \omega_{i j, k}^{2}
\end{aligned}
$$




$$
\begin{aligned}
& \times \vec{\Gamma}\left(\mathcal{X}_{j, k}+\hat{x}_{j, k \mid k-\tau_{i j}} \hat{x}_{j, k \mid k-\tau_{i j}}^{T}\right) \\
& +N \bar{\gamma} \sum_{j=1}^{N} \omega_{i j, k}^{2} \lambda_{\max }\left(\mathcal{X}_{j, k}\right) I+\mathcal{D}_{i, k} \mathcal{Q}_{i, k}^{\zeta} \mathcal{D}_{i, k}^{T} \\
& +\left(\mathcal{K}_{i, k}-\left(1+\mu_{i, k}\right)\left(\mathcal{A}_{i, k}+\omega_{i i, k} \vec{\Gamma}\right) \mathcal{T}_{i, k} \mathcal{C}_{i, k}^{T} \bar{\Psi}_{i, k}^{T}\right. \\
& \left.\times \Xi_{i, k}^{-1}\right) \Xi_{i, k}\left(\mathcal{K}_{i, k}-\left(1+\mu_{i, k}\right)\left(\mathcal{A}_{i, k}+\omega_{i i, k} \vec{\Gamma}\right)\right. \\
& \left.\times \mathcal{T}_{i, k} \mathcal{C}_{i, k}^{T} \bar{\Psi}_{i, k}^{T} \Xi_{i, k}^{-1}\right)^{T}-\left(1+\mu_{i, k}\right)^{2}\left(\mathcal{A}_{i, k}+\omega_{i i, k} \vec{\Gamma}\right) \\
& \times \mathcal{T}_{i, k} \mathcal{C}_{i, k}^{T} \bar{\Psi}_{i, k}^{T} \Xi_{i, k}^{-1} \bar{\Psi}_{i, k} \mathcal{C}_{i, k} \mathcal{T}_{i, k}\left(\mathcal{A}_{i, k}+\omega_{i i, k} \vec{\Gamma}\right)^{T},
\end{aligned}
$$

where

$$
\begin{aligned}
\Xi_{i, k}= & \left(1+\mu_{i, k}\right) \bar{\Psi}_{i, k} \mathcal{C}_{i, k} \mathcal{T}_{i, k} \mathcal{C}_{i, k}^{T} \bar{\Psi}_{i, k}^{T}+\mathcal{Q}_{i, k}^{\eta} \\
& +\breve{\Psi}_{i, k} \circ\left(\mathcal{C}_{i, k} \mathcal{X}_{i, k} \mathcal{C}_{i, k}^{T}\right) .
\end{aligned}
$$

Next, let $\mathcal{K}_{i, k}$

$$
\mathcal{K}_{i, k} \triangleq\left[\left(1+\mu_{i, k}\right)\left(\mathcal{A}_{i, k}+\omega_{i i, k} \vec{\Gamma}\right) \mathcal{T}_{i, k} \mathcal{C}_{i, k}^{T} \bar{\Psi}_{i, k}^{T}\right] \Xi_{i, k}^{-1} .
$$

Then, we have the locally minimized upper bound $\mathcal{T}_{i, k+1}$ denoted by

$$
\begin{aligned}
& \mathcal{T}_{i, k+1} \\
= & \left(1+\mu_{i, k}\right)\left(\mathcal{A}_{i, k}+\omega_{i i, k} \vec{\Gamma}\right) \mathcal{T}_{i, k}\left(\mathcal{A}_{i, k}+\omega_{i i, k} \vec{\Gamma}\right)^{T} \\
& +2\left(1+\mu_{i, k}^{-1}\right)(N-1) \sum_{j=1, j \neq i}^{N} \omega_{i j, k}^{2} \\
& \times \vec{\Gamma}\left(\mathcal{X}_{j, k}+\hat{x}_{j, k \mid k-\tau_{i j}} \hat{x}_{j, k \mid k-\tau_{i j}}^{T}\right) \\
& +N \bar{\gamma} \sum_{j=1}^{N} \omega_{i j, k}^{2} \lambda_{\max }\left(\mathcal{X}_{j, k}\right) I+\mathcal{D}_{i, k} \mathcal{Q}_{i, k}^{\zeta} \mathcal{D}_{i, k}^{T} \\
& -\left(1+\mu_{i, k}\right)^{2}\left(\mathcal{A}_{i, k}+\omega_{i i, k} \vec{\Gamma}\right) \mathcal{T}_{i, k} \mathcal{C}_{i, k}^{T} \bar{\Psi}_{i, k}^{T} \Xi_{i, k}^{-1} \\
& \times \bar{\Psi}_{i, k} \mathcal{C}_{i, k} \mathcal{T}_{i, k}\left(\mathcal{A}_{i, k}+\omega_{i i, k} \vec{\Gamma}\right)^{T},
\end{aligned}
$$

which completes the proof of this theorem.

Remark 4: So far, a new optimized estimation scheme has been given for DTVCNs subject to NIIOs and dynamical bias. To be more specific, both the communication delays and fading observations have been adequately taken into account, where a predictive-based mechanism has been proposed to compensate the potential influences caused by the communication delays. Subsequently, both the predictive-based estimation information and available probability of fading observations have been actively introduced to design a new recursive state estimator. It should be noticed that some positive scalars $\mu_{i, k}$ have been introduced in Theorem 3. In the subsequent estimation algorithm implementation, the parameters $\mu_{i, k}$ can be fixed and then adjusted to enhance the solvability of the proposed DCBSE algorithm. Compared with the conventional Kalman estimation algorithm, the newly proposed DCBSE algorithm is a locally optimal one, where a locally minimized upper bound with regards to the estimation error covariance matrix has been found and the desirable estimator gain matrix has been parameterized in terms of the solution to an RME. The major reason lies in that it is impossible to obtain the exact value of the estimation error covariance matrix due to the existence of NIIOs. As such, an alternative estimation approach has been proposed to achieve admissible estimation requirements.
For the ease of the implementation, the following DCBSE algorithm based on the predictive scheme is outlined, where the parameter selection and updating way are summarized.

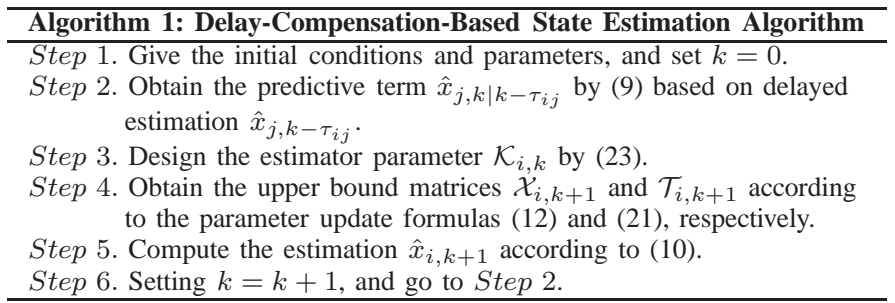

Remark 5: As illustrated in Algorithm 1, based on (9) and the RME in (21), the predictive estimation term $\hat{x}_{j, k \mid k-\tau_{i j}}$ and the estimator parameter $\mathcal{K}_{i, k}$ can be obtained easily. Then, the addressed DCBSE problem for DTVCNs with NIIOs and dynamical bias is solvable. Note that the desirable parameters are available via testifying the feasibility of some forward matrix difference equations. Consequently, the developed DCBSE scheme within time-varying framework is satisfactory for the requirement of online applications.

\section{Performance Analysis of Estimation Method}

In this section, the algorithm performance analysis is conducted, where the monotonicity of the new DCBSE scheme is shown.

For ease of illustration, let the random variables $\psi_{i t, k}$ $\left(t=1,2, \cdots, n_{y}\right)$ be $\psi_{i, k}$ with $\mathbb{E}\left\{\psi_{i, k}\right\}=\bar{\psi}_{i, k}$ and $\mathbb{E}\left\{\left(\psi_{i, k}-\bar{\psi}_{i, k}\right)\left(\psi_{i, k}-\bar{\psi}_{i, k}\right)^{T}\right\}=\breve{\psi}_{i, k}$. Moreover, the relationship between $\bar{\psi}_{i, k}$ and $\operatorname{tr}\left(\mathcal{T}_{i, k+1}\right)$ is discussed as $\breve{\psi}_{i, k}$ is fixed. To further provide the monotonicity analyses, the following lemma is helpful.

Lemma 1: For the matrices $A, B, X$ as well as $Y$ with proper dimensions and the variable $t \in \mathbb{R}$, one has

$$
\begin{aligned}
& \frac{\mathrm{d}}{\mathrm{d} t} \operatorname{tr}(X+t Y)=\operatorname{tr}(Y), \\
& \frac{\mathrm{d}}{\mathrm{d} t} \operatorname{tr}\left[B^{T}(X+t Y)^{-1} A\right] \\
= & -\operatorname{tr}\left[B^{T}(X+t Y)^{-1} Y(X+t Y)^{-1} A\right] .
\end{aligned}
$$

Theorem 4: If the occurrence probability $\bar{\psi}_{i, k}$ increases, it is observed that $\operatorname{tr}\left(\mathcal{T}_{i, k+1}\right)$ is non-increasing.

Proof: Notice that $\Xi_{i, k}$ in (24) and $\mathcal{T}_{i, k+1}$ in (32) contain the scalar $\bar{\psi}_{i, k}$. Then, it follows from 1 that

$$
\begin{aligned}
& \frac{\mathrm{d} \operatorname{tr}\left\{\mathcal{T}_{i, k+1}\right\}}{\mathrm{d} \bar{\psi}_{i, k}} \\
= & \frac{\mathrm{d} \operatorname{tr}}{\mathrm{d} \bar{\psi}_{i, k}}\left\{\left(1+\mu_{i, k}\right)\left(\mathcal{A}_{i, k}+\omega_{i i, k} \vec{\Gamma}\right) \mathcal{T}_{i, k}\left(\mathcal{A}_{i, k}+\omega_{i i, k} \vec{\Gamma}\right)^{T}\right. \\
& +2\left(1+\mu_{i, k}^{-1}\right)(N-1) \sum_{j=1, j \neq i}^{N} \omega_{i j, k}^{2} \\
& \times \vec{\Gamma}\left(\mathcal{X}_{j, k}+\hat{x}_{j, k \mid k-\tau_{i j}} \hat{x}_{j, k \mid k-\tau_{i j}}^{T}\right) \\
& +N \bar{\gamma} \sum_{j=1}^{N} \omega_{i j, k}^{2} \lambda_{\max }\left(\mathcal{X}_{j, k}\right) I+\mathcal{D}_{i, k} \mathcal{Q}_{i, k}^{\zeta} \mathcal{D}_{i, k}^{T} \\
& -\left(1+\mu_{i, k}\right)^{2} \bar{\psi}_{i, k}^{2}\left(\mathcal{A}_{i, k}+\omega_{i i, k} \vec{\Gamma}\right) \mathcal{T}_{i, k} \mathcal{C}_{i, k}^{T} \Xi_{i, k}^{-1}
\end{aligned}
$$




$$
\begin{aligned}
& \left.\times \mathcal{C}_{i, k} \mathcal{T}_{i, k}\left(\mathcal{A}_{i, k}+\omega_{i i, k} \vec{\Gamma}\right)^{T}\right\} \\
= & \operatorname{tr}\left\{-2\left(1+\mu_{i, k}\right)^{2} \bar{\psi}_{i, k}\left(\mathcal{A}_{i, k}+\omega_{i i, k} \vec{\Gamma}\right) \mathcal{T}_{i, k} \mathcal{C}_{i, k}^{T} \Xi_{i, k}^{-1}\right. \\
& \times \mathcal{C}_{i, k} \mathcal{T}_{i, k}\left(\mathcal{A}_{i, k}+\omega_{i i, k} \vec{\Gamma}\right)^{T} \\
& +2\left(1+\mu_{i, k}\right)^{2} \bar{\psi}_{i, k}^{2}\left(\mathcal{A}_{i, k}+\omega_{i i, k} \vec{\Gamma}\right) \mathcal{T}_{i, k} \mathcal{C}_{i, k}^{T} \Xi_{i, k}^{-1} \\
& \times\left[\left(1+\mu_{i, k}\right) \bar{\psi}_{i, k} \mathcal{C}_{i, k} \mathcal{T}_{i, k} \mathcal{C}_{i, k}^{T}\right] \\
& \left.\times \Xi_{i, k}^{-1} \mathcal{C}_{i, k} \mathcal{T}_{i, k}\left(\mathcal{A}_{i, k}+\omega_{i i, k} \vec{\Gamma}\right)^{T}\right\} .
\end{aligned}
$$

Adding some zero terms to the above equation, one has

$$
\begin{aligned}
& \frac{\operatorname{dtr}\left\{\mathcal{T}_{i, k+1}\right\}}{\mathrm{d} \bar{\psi}_{i, k}} \\
= & \operatorname{tr}\left\{-2\left(1+\mu_{i, k}\right)^{2} \bar{\psi}_{i, k}\left(\mathcal{A}_{i, k}+\omega_{i i, k} \vec{\Gamma}\right) \mathcal{T}_{i, k} \mathcal{C}_{i, k}^{T} \Xi_{i, k}^{-1}\right. \\
& \times \mathcal{C}_{i, k} \mathcal{T}_{i, k}\left(\mathcal{A}_{i, k}+\omega_{i i, k} \vec{\Gamma}\right)^{T} \\
& +2\left(1+\mu_{i, k}\right)^{2} \bar{\psi}_{i, k}\left(\mathcal{A}_{i, k}+\omega_{i i, k} \vec{\Gamma}\right) \mathcal{T}_{i, k} \mathcal{C}_{i, k}^{T} \Xi_{i, k}^{-1} \\
& \times\left[\left(1+\mu_{i, k}\right) \bar{\psi}_{i, k}^{2} \mathcal{C}_{i, k} \mathcal{T}_{i, k} \mathcal{C}_{i, k}^{T}+\mathcal{Q}_{i, k}^{\eta}\right. \\
& \left.+\breve{\Psi}_{i, k} \circ\left(\mathcal{C}_{i, k} \mathcal{X}_{i, k} \mathcal{C}_{i, k}^{T}\right)\right] \Xi_{i, k}^{-1} \mathcal{C}_{i, k} \mathcal{T}_{i, k}\left(\mathcal{A}_{i, k}+\omega_{i i, k} \vec{\Gamma}\right)^{T} \\
& -2\left(1+\mu_{i, k}\right)^{2} \bar{\psi}_{i, k}\left(\mathcal{A}_{i, k}+\omega_{i i, k} \vec{\Gamma}\right) \mathcal{T}_{i, k} \mathcal{C}_{i, k}^{T} \Xi_{i, k}^{-1} \\
& \times\left[\mathcal{Q}_{i, k}^{\eta}+\breve{\Psi}_{i, k} \circ\left(\mathcal{C}_{i, k} \mathcal{X}_{i, k} \mathcal{C}_{i, k}^{T}\right)\right] \\
& \left.\times \Xi_{i, k}^{-1} \mathcal{C}_{i, k} \mathcal{T}_{i, k}\left(\mathcal{A}_{i, k}+\omega_{i i, k} \vec{\Gamma}\right)^{T}\right\} .
\end{aligned}
$$

After the simple algebraic calculation, we arrive at

$$
\begin{aligned}
& \frac{\mathrm{d} \operatorname{tr}\left\{\mathcal{T}_{i, k+1}\right\}}{\mathrm{d} \bar{\psi}_{i, k}} \\
= & \operatorname{tr}\left\{-2\left(1+\mu_{i, k}\right)^{2} \bar{\psi}_{i, k}\left(\mathcal{A}_{i, k}+\omega_{i i, k} \vec{\Gamma}\right) \mathcal{T}_{i, k} \mathcal{C}_{i, k}^{T} \Xi_{i, k}^{-1}\right. \\
& \times\left[\mathcal{Q}_{i, k}^{\eta}+\breve{\Psi}_{i, k} \circ\left(\mathcal{C}_{i, k} \mathcal{X}_{i, k} \mathcal{C}_{i, k}^{T}\right)\right] \Xi_{i, k}^{-1} \mathcal{C}_{i, k} \mathcal{T}_{i, k} \\
& \left.\times\left(\mathcal{A}_{i, k}+\omega_{i i, k} \vec{\Gamma}\right)^{T}\right\},
\end{aligned}
$$

which leads to

$$
\frac{\mathrm{d} \operatorname{tr}\left\{\mathcal{T}_{i, k+1}\right\}}{\mathrm{d} \bar{\psi}_{i, k}} \leq 0 .
$$

Consequently, it is concluded that $\operatorname{tr}\left\{\mathcal{T}_{i, k+1}\right\}$ is non-increasing when $\bar{\psi}_{i, k}$ increases.

As a special case, the same assertion can be obtained when $\psi_{i, k}$ is governed by the Bernoulli distribution, which is shown in the following corollary.

Corollary 1: If $\psi_{i, k}$ is a Bernoulli distributed random variable and its mean $\bar{\psi}_{i, k}$ increases, we can also obtain the fact that $\operatorname{tr}\left(\mathcal{T}_{i, k+1}\right)$ is non-increasing.

Proof: In this case, we have $\bar{\Psi}_{i, k}=\bar{\psi}_{i, k} I$ and $\breve{\Psi}_{i, k}=$ $\bar{\psi}_{i, k}\left(1-\bar{\psi}_{i, k}\right) I$. Along the same line of Theorem 4, one has

$$
\begin{aligned}
& \frac{\mathrm{d} \operatorname{tr}\left\{\mathcal{T}_{i, k+1}\right\}}{\mathrm{d} \bar{\psi}_{i, k}} \\
= & \operatorname{tr}\left\{-2\left(1+\mu_{i, k}\right)^{2} \bar{\psi}_{i, k}\left(\mathcal{A}_{i, k}+\omega_{i i, k} \vec{\Gamma}\right) \mathcal{T}_{i, k} \mathcal{C}_{i, k}^{T} \Xi_{i, k}^{-1}\right. \\
& \times \mathcal{C}_{i, k} \mathcal{T}_{i, k}\left(\mathcal{A}_{i, k}+\omega_{i i, k} \vec{\Gamma}\right)^{T} \\
& +\left(1+\mu_{i, k}\right)^{2} \bar{\psi}_{i, k}^{2}\left(\mathcal{A}_{i, k}+\omega_{i i, k} \vec{\Gamma}\right) \mathcal{T}_{i, k} \mathcal{C}_{i, k}^{T} \Xi_{i, k}^{-1} \\
& \times\left[2\left(1+\mu_{i, k}\right) \bar{\psi}_{i, k} \mathcal{C}_{i, k} \mathcal{T}_{i, k} \mathcal{C}_{i, k}^{T}+\left(1-2 \bar{\psi}_{i, k}\right) I\right. \\
& \left.\left.\circ\left(\mathcal{C}_{i, k} \mathcal{X}_{i, k} \mathcal{C}_{i, k}^{T}\right)\right] \Xi_{i, k}^{-1} \mathcal{C}_{i, k} \mathcal{T}_{i, k}\left(\mathcal{A}_{i, k}+\omega_{i i, k} \vec{\Gamma}\right)^{T}\right\}
\end{aligned}
$$

$$
\begin{aligned}
\leq & \operatorname{tr}\left\{-2\left(1+\mu_{i, k}\right)^{2} \bar{\psi}_{i, k}\left(\mathcal{A}_{i, k}+\omega_{i i, k} \vec{\Gamma}\right) \mathcal{T}_{i, k} \mathcal{C}_{i, k}^{T} \Xi_{i, k}^{-1}\right. \\
& \times \mathcal{C}_{i, k} \mathcal{T}_{i, k}\left(\mathcal{A}_{i, k}+\omega_{i i, k} \vec{\Gamma}\right)^{T} \\
& +\left(1+\mu_{i, k}\right)^{2} \bar{\psi}_{i, k}\left(\mathcal{A}_{i, k}+\omega_{i i, k} \vec{\Gamma}\right) \mathcal{T}_{i, k} \mathcal{C}_{i, k}^{T} \Xi_{i, k}^{-1} \\
& \times\left[2\left(1+\mu_{i, k}\right) \bar{\psi}_{i, k}^{2} \mathcal{C}_{i, k} \mathcal{T}_{i, k} \mathcal{C}_{i, k}^{T}+2 \bar{\psi}_{i, k}\right. \\
& \times\left(1-\bar{\psi}_{i, k}\right) I \circ\left(\mathcal{C}_{i, k} \mathcal{X}_{i, k} \mathcal{C}_{i, k}^{T}\right)+2 \mathcal{Q}_{i, k}^{\eta} \\
& \left.\left.-\bar{\psi}_{i, k} I \circ\left(\mathcal{C}_{i, k} \mathcal{X}_{i, k} \mathcal{C}_{i, k}^{T}\right)\right] \Xi_{i, k}^{-1} \mathcal{C}_{i, k} \mathcal{T}_{i, k}\left(\mathcal{A}_{i, k}+\omega_{i i, k} \vec{\Gamma}\right)^{T}\right\} \\
= & \operatorname{tr}\left\{-\left(1+\mu_{i, k}\right)^{2} \bar{\psi}_{i, k}\left(\mathcal{A}_{i, k}+\omega_{i i, k} \vec{\Gamma}\right) \mathcal{T}_{i, k} \mathcal{C}_{i, k}^{T} \Xi_{i, k}^{-1}\right. \\
& \left.\times\left[\bar{\psi}_{i, k} \circ\left(\mathcal{C}_{i, k} \mathcal{X}_{i, k} \mathcal{C}_{i, k}^{T}\right)\right] \Xi_{i, k}^{-1} \mathcal{C}_{i, k} \mathcal{T}_{i, k}\left(\mathcal{A}_{i, k}+\omega_{i i, k} \vec{\Gamma}\right)^{T}\right\} \\
\leq & 0 .
\end{aligned}
$$

As such, it is concluded that $\operatorname{tr}\left\{\mathcal{T}_{i, k+1}\right\}$ is non-increasing when $\bar{\psi}_{i, k}$ increases.

Remark 6: Compared with some existing estimation results, this paper proposes a mathematical analysis method to discuss the monotonicity performance of the developed DCBSE algorithm with regard to the fading observations. Particularly, in Theorem 4 and Corollary 1, the theoretical proofs have been provided to reveal the monotonicity relationship between the fading probability and the estimation accuracy. As shown in the above analyses, it follows that the estimation accuracy becomes worse when the fading observations are severe. That is to say, the estimation error is larger if less measurement information can be utilized in the estimator side, which is indeed consistent with the essential intuition in the practical engineering.

\section{An Illustrative Simulation}

In this section, the following simulation experiment is conducted and the usefulness of newly DCBSE scheme is discussed.

For the DTVCNs (1)-(2), consider the following related matrix parameters:

$$
\begin{aligned}
A_{1, k} & =\left[\begin{array}{cc}
0.35+0.35 \cos (k) & 0.52 \\
-1.38 & 1.55
\end{array}\right], \\
A_{2, k} & =\left[\begin{array}{cc}
0.65 & 0.5 \\
-0.05 & -0.75
\end{array}\right], \\
A_{3, k} & =\left[\begin{array}{cc}
0.86 & 0.72+0.3 \sin (k) \\
-0.25 & 0.84
\end{array}\right] \\
D_{1, k} & =\left[\begin{array}{cc}
0.31 \\
0.32
\end{array}\right], D_{2, k}=\left[\begin{array}{l}
0.26 \\
0.22
\end{array}\right], D_{3, k}=\left[\begin{array}{l}
0.38 \\
0.40
\end{array}\right], \\
B_{1, k} & =\left[\begin{array}{cc}
1.1 & 0.9 \\
0.43 & -0.91
\end{array}\right], B_{2, k}=\left[\begin{array}{ll}
0.65 & 0.98 \\
0.37 & 0.56
\end{array}\right], \\
B_{3, k} & =\left[\begin{array}{cc}
0.52 & -0.19 \\
1.31 & 0.38
\end{array}\right], C_{1, k}=\left[\begin{array}{ll}
1.6 & 1.5
\end{array}\right], \\
C_{2, k} & =\left[\begin{array}{cc}
1.7 & 1.9
\end{array}\right], C_{3, k}=\left[\begin{array}{ll}
1.8 & 1.5
\end{array}\right], \\
F_{1, k} & =\left[\begin{array}{cc}
0.51 & 0.78 \\
-0.3 & 0.6
\end{array}\right], F_{2, k}=\left[\begin{array}{cc}
0.46 & 0.48 \\
0.4 & 0.36
\end{array}\right], \\
F_{3, k} & =\left[\begin{array}{cc}
0.49 & 0.58 \\
0.63 & -0.16
\end{array}\right] .
\end{aligned}
$$

Moreover, the other parameters are chose by $\bar{\Gamma}=$ $\operatorname{diag}\{0.25,0.25\}, \bar{\gamma}=0.01, w_{i j, k}=0.5(i \neq j)$ and $w_{i i, k}=1$ $(i=1,2,3)$. 
During the simulation experiment, choose the initial conditions as $\bar{x}_{1,0}=\left[\begin{array}{ll}2 & 1\end{array}\right]^{T}, \bar{x}_{2,0}=\left[\begin{array}{ll}3 & 1\end{array}\right]^{T}, \bar{x}_{3,0}=\left[\begin{array}{ll}1 & -4\end{array}\right]^{T}$, $\bar{b}_{1,0}=\left[\begin{array}{ll}1 & 1\end{array}\right]^{T}, \bar{b}_{2,0}=\left[\begin{array}{ll}1 & 1\end{array}\right]^{T}, \bar{b}_{3,0}=\left[\begin{array}{ll}-1 & -1\end{array}\right]^{T}, \mathcal{T}_{i, 0}=5 I$, $\mathcal{X}_{i, 0}=\overrightarrow{\vec{x}}_{i, 0} \overrightarrow{\vec{x}}_{i, 0}^{T}$ with $\vec{x}_{i, 0}=\left[\begin{array}{l}\bar{x}_{i, 0} \\ \bar{b}_{i, 0}\end{array}\right], \epsilon_{i, k}=0.5, \mu_{i, k}=1.5$, $Q_{i, k}^{\beta}=0.15 I, Q_{i, k}^{\theta}=0.25, Q_{i, k}^{\eta}=0.2(i=1,2,3)$, $\hat{x}_{i, 0}=\left[\begin{array}{llll}1 & 1 & 1 & 1\end{array}\right]^{T}$, and $\hat{x}_{i, k}=\left[\begin{array}{lllll}0.2 & 0.2 & 0.2 & 0.2\end{array}\right]^{T}(k<0)$ $(i=1,2,3)$. In order to depict the estimation performance, MSE $i$ denotes the mean square error (MSE) for the estimation of $x_{i, k}$ and is calculated by $\frac{1}{M} \sum_{s=1}^{M}\left(x_{i, k}^{l(s)}-\hat{x}_{i, k}^{l(s)}\right)^{2}$, where $M=1000$ is the run number of the simulation test and $x_{i, k}^{l}(l=1,2 ; i=1,2,3)$ denotes the $l$-th element of $x_{i, k}$. Moreover, the sum of the MSE $i(i=1,2,3)$ is denoted by SMSE.

To validate the efficiency and advantages of the proposed DCBSE algorithm, we compare the cases with/without prediction compensation, i.e., Case I: the state estimation under the DCBSE algorithm; Case II: the state estimation without the updating rule (9). Accordingly, the simulation results under $a_{i t}=0.5, b_{i t}=1$ and $\tau_{i j}=5$ are presented in Figs. 15, where Figs. 1-3 plot the state trajectories of 3 nodes and their estimations with/without prediction compensation. Fig. 4 depicts the $\log (\mathrm{MSE} i)$ of 3 nodes and their upper bounds under Case I. The comparisons of SMSE with/without delay compensation under 1000 iterations are shown in Fig. 5. It is observed from the simulations that the new DCBSE algorithm in Case I performs well than the one as in Case II. The major reason lies in that the predictive compensation mechanism has been introduced to attenuate the influences induced by the communication delays, thereby improving the estimation accuracy.
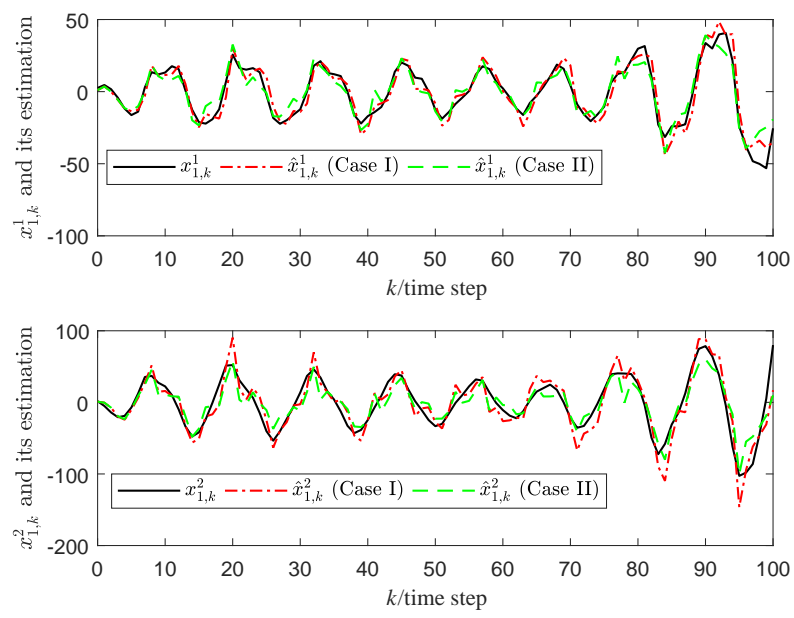

Fig. 1. The curves of $x_{1, k}$ and $\hat{x}_{1, k}$.

In order to further reveal the estimation algorithm performance, we conduct more experiments to discuss the relationship between SMSE and different situations of the fading observations. For the comparison purpose, another three cases are taken into account, i.e., Case a: $a_{i t}=0, b_{i t}=1 / 3$, Case b: $a_{i t}=1 / 3, b_{i t}=2 / 3$, Case c: $a_{i t}=2 / 3, b_{i t}=1$. For the above mentioned cases, it can be observed that the fading
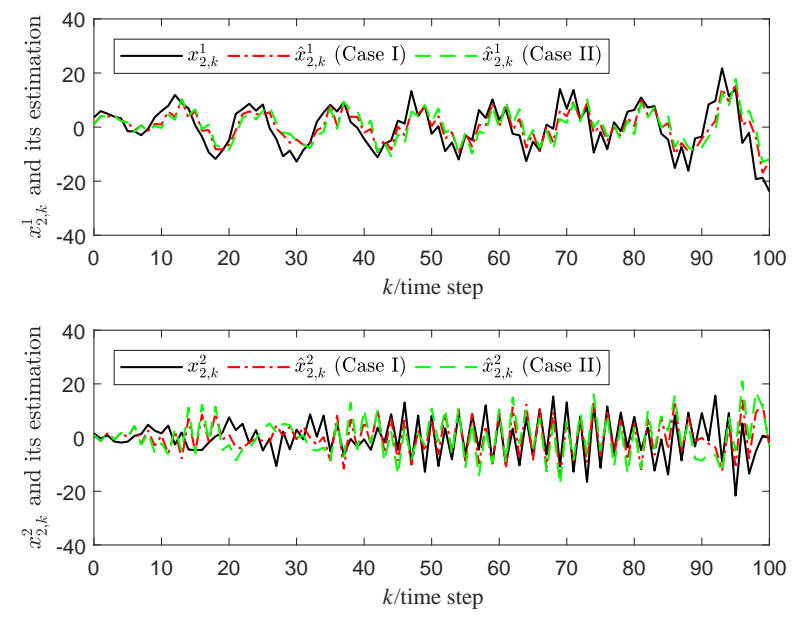

Fig. 2. The curves of $x_{2, k}$ and $\hat{x}_{2, k}$.
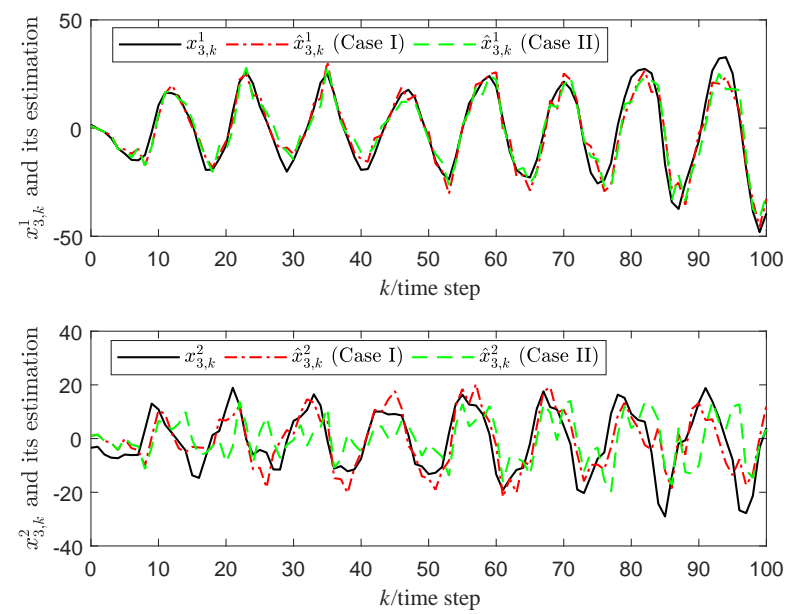

Fig. 3. The curves of $x_{3, k}$ and $\hat{x}_{3, k}$.
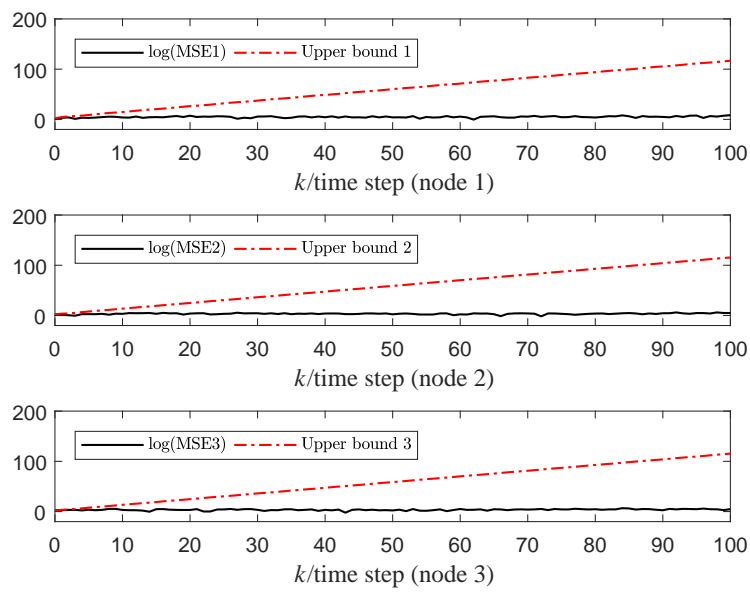

Fig. 4. $\log (\mathrm{MSE} i)$ and upper bounds.

observations are most severe in Case a. Based on the main 


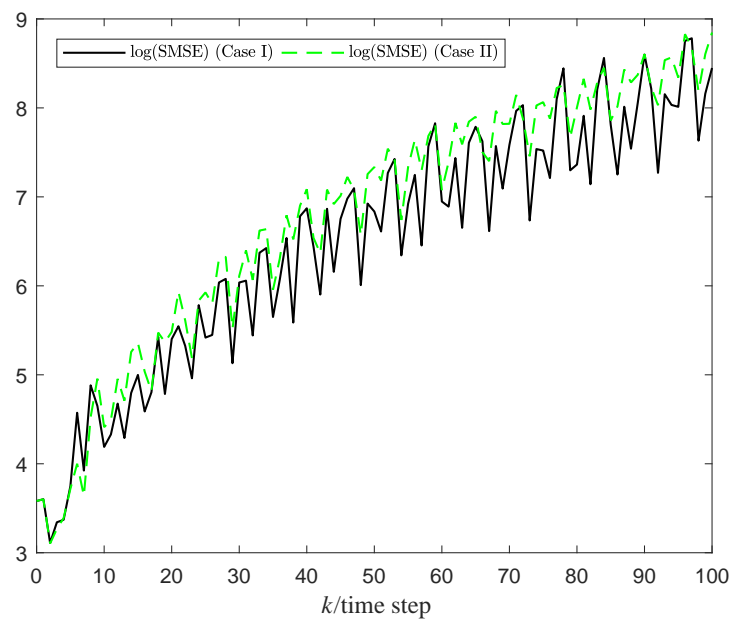

Fig. 5. $\log$ (SMSE) with/without compensation (1000 iterations).

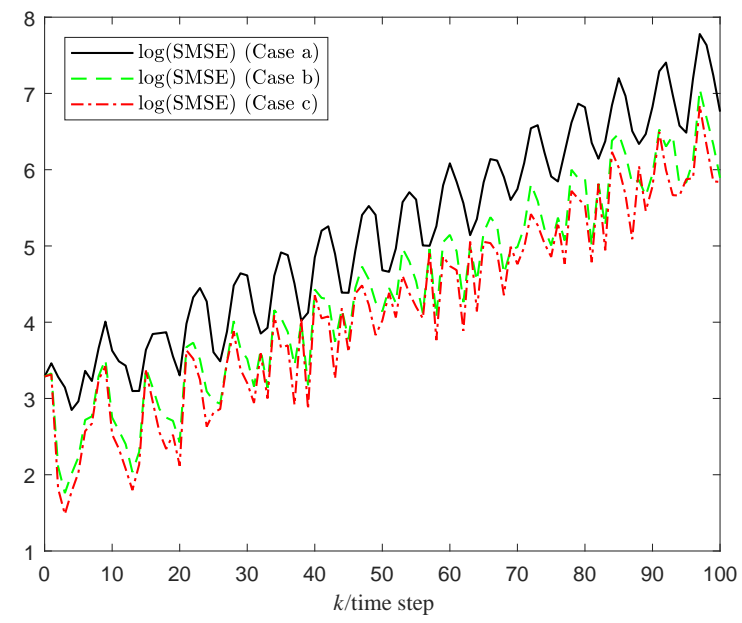

Fig. 6. $\log$ (SMSE) with different fading observations (1000 iterations).



Fig. 7. $\log (\mathrm{SMSE})$ with different $Q_{i, k}^{\beta}$ (1000 iterations).

results with related parameters, the developed DCBSE strategy in Algorithm 1 can be implemented. Accordingly, the relationship between $\log$ (SMSE) and different fading observations is plotted in Fig. 6 and the $\log \left(\operatorname{tr}\left\{\mathcal{T}_{1, k+1}\right\}\right)$ under three cases are listed in Tables I-III, which further show that the estimation error becomes larger when the fading observations are severe as in Case a. This is consistent with the practical viewpoint because less information is available in the remote estimator side in Case a, hence the estimation performance becomes worse compared with those in Case b and Case c.

TABLE I

$\log \left(\operatorname{tr}\left\{\mathcal{T}_{1, k+1}\right\}\right)($ node 1$)$

\begin{tabular}{cccccccc}
\hline time $(k)$ & $\cdots$ & 65 & 66 & 67 & 68 & 69 & $\cdots$ \\
\hline Case a & $\cdots$ & 76.358 & 77.267 & 78.354 & 79.627 & 80.994 & $\cdots$ \\
\hline Case b & $\cdots$ & 76.038 & 77.017 & 78.17 & 79.448 & 80.763 & $\cdots$ \\
\hline Case c & $\cdots$ & 75.984 & 76.977 & 78.14 & 79.416 & 80.717 & $\cdots$ \\
\hline
\end{tabular}

TABLE II

$\log \left(\operatorname{tr}\left\{\mathcal{T}_{2, k+1}\right\}\right)$ (node 2$)$

\begin{tabular}{cccccccc}
\hline time $(k)$ & $\cdots$ & 65 & 66 & 67 & 68 & 69 & $\cdots$ \\
\hline Case a & $\cdots$ & 74.944 & 76.172 & 77.349 & 78.461 & 79.542 & $\cdots$ \\
\hline Case b & $\cdots$ & 74.767 & 76.018 & 77.189 & 78.281 & 79.347 & $\cdots$ \\
\hline Case c & $\cdots$ & 74.748 & 76.002 & 77.172 & 78.262 & 79.326 & $\cdots$ \\
\hline
\end{tabular}

TABLE III

$\log \left(\operatorname{tr}\left\{\mathcal{T}_{3, k+1}\right\}\right)$ (node 3 )

\begin{tabular}{cccccccc}
\hline time $(k)$ & $\cdots$ & 65 & 66 & 67 & 68 & 69 & $\cdots$ \\
\hline Case a & $\cdots$ & 74.876 & 76.118 & 77.307 & 78.401 & 79.44 & $\cdots$ \\
\hline Case b & $\cdots$ & 74.656 & 75.929 & 77.125 & 78.213 & 79.243 & $\cdots$ \\
\hline Case c & $\cdots$ & 74.624 & 75.901 & 77.098 & 78.187 & 79.217 & $\cdots$ \\
\hline
\end{tabular}

For the comparison purpose, the covariance matrices of random bias with $Q_{i, k}^{\beta}=0.15$ and $Q_{i, k}^{\beta}=0.65$ are considered, where the effect caused by the dynamical bias is discussed. Subsequently, Fig. 7 depicts the $\log (\mathrm{SMSE})$ in different bias cases under 1000 iterations, which shows that the estimation error is relatively larger when $Q_{i, k}^{\beta}=0.65$. That is to say, the estimation accuracy becomes worse when the random bias is severe. Overall, the above comparisons reflect the estimation performance of proposed DCBSE method and further illustrate the usefulness of main results.

\section{CONClusions}

In this paper, we have made one of the first attempts to handle the DCBSE problem for DTVCNs subject to NIIOs and dynamical bias. By fully taking the available information of communication delays and fading observations, a simultaneous bias and state estimation scheme has been proposed in terms of the delay-prediction-compensation approach. The major features of the proposed DCBSE strategy include: 1) the NIIOs have been actively compensated during the estimation algorithm design by properly employing the available information of NIIOs; 2) a new estimation method of the recursive manner has been presented, where the expression form of the estimator gain matrix and the illustration of the proposed DCBSE algorithm have been given; and 3) the monotonicity proofs with regard to the fading observations characterized by 
different probability distribution have been provided. Finally, some simulation experiments have been proposed, where the estimation performance comparisons with/without the predictive compensation mechanism have been demonstrated and the advantages of main results have been illustrated. Further research extensions include the design of DCBSE method for DTVCNs subject to communication protocols in order to save the limited network resources. Moreover, it is interesting to deal with the boudedness analysis issue of the proposed estimation strategy, which can be expected in the near future.

\section{REFERENCES}

[1] M. S. Ali, M. Usha, Z. Orman, and S. Arik, Improved result on state estimation for complex dynamical networks with time varying delays and stochastic sampling via sampled-data control, Neural Networks, vol. 114, pp. 28-37, 2019.

[2] Y. Alipouri and B. Huang, Distributed multiple step ahead prediction considering communication delays, IET Control Theory and Applications, vol. 13, no. 6, pp. 806-814, 2019.

[3] M. T. Asif, N. Mitrovic, J. Dauwels, and P. Jaillet, Matrix and tensor based methods for missing data estimation in large traffic networks, IEEE Transactions on Intelligent Transportation Systems, vol. 17, no. 7, pp. 1816-1825, 2016.

[4] Y. Chen and Z. Wang, Local stabilization for discrete-time systems with distributed state delay and fast-varying input delay under actuator saturations, IEEE Transactions on Automatic Control, in press, DOI: 10.1109/TAC.2020.2991013.

[5] D. Chen, W. Chen, J. Hu, and H. Liu, Variance-constrained filtering for discrete-time genetic regulatory networks with state delay and random measurement delay, International Journal of Systems Science, vol. 50, no. 2, pp. 231-243, 2019.

[6] Y. Chen, Z. Wang, L. Wang and W. Sheng, Mixed $H_{2} / H_{\infty}$ state estimation for discrete-time switched complex networks with random coupling strengths through redundant channels, IEEE Transactions on Neural Networks and Learning Systems, vol. 31, no. 10, pp. 4130-4142, 2020.

[7] Y. Chen, Z. Wang, J. Hu and Q.-L. Han, Synchronization control for discrete-time delayed dynamical networks with switching topology under actuator saturations, IEEE Transactions on Neural Networks and Learning Systems, in press, DOI: 10.1109/TNNLS.2020.2996094.

[8] Y. Chen, Z. Wang, Y. Yuan and P. Date, Distributed $H_{\infty}$ filtering for switched stochastic delayed systems over sensor networks with fading measurements, IEEE Transactions on Cybernetics, vol. 50, no. 1, pp. 2 14, Jan. 2020.

[9] D. Ding, Z. Wang, and Q.-L. Han, A set-membership approach to eventtriggered filtering for general nonlinear systems over sensor networks, IEEE Transactions on Automatic Control, vol. 65, no. 4, pp. 1792-1799, 2020.

[10] H. Dong, N. Hou, and Z. Wang, Fault estimation for complex networks with randomly varying topologies and stochastic inner couplings, Automatica, vol. 112, Article No: 108734, 2020. DOI: 10.1016/j.automatica.2019.108734.

[11] H. Dong, N. Hou, Z. Wang, and W. Ren, Variance-constrained state estimation for complex networks with randomly varying topologies, IEEE Transactions on Neural Networks and Learning Systems, vol. 29, no. 7, pp. 2757-2768, 2018.

[12] G. Duan, F. Xiao, and L. Wang, Asynchronous periodic edge-event triggered control for double-integrator networks with communication time delays, IEEE Transactions on Cybernetics, vol. 48, no. 2, pp. 675688,2018

[13] N. Hou, Z. Wang, D. W. C. Ho and H. Dong, Robust partial-nodesbased state estimation for complex networks under deception attacks, IEEE Transactions on Neural Networks and Learning Systems, vol. 31, no. 6, pp. 2793-2802, Jun. 2020.

[14] C. $\mathrm{Hu}, \mathrm{H}$. He, and H. Jiang, Synchronization of complex-valued dynamic networks with intermittently adaptive coupling: A direct error method, Automatica, vol. 112, Article No: 108675, 2020. DOI: 10.1016/j.automatica.2019.108675.

[15] J. Hu, Z. Wang, and H. Gao, Joint state and fault estimation for uncertain time-varying nonlinear systems with randomly occurring faults and sensor saturations, Automatica, vol. 97, pp. 150-160, 2018.
[16] J. Hu, Z. Wang, F. E. Alsaadi, and T. Hayat, Event-based filtering for time-varying nonlinear systems subject to multiple missing measurements with uncertain missing probabilities, Information Fusion, vol. 38, no. 74-83, 2017.

[17] J. Hu, Z. Wang, G.-P. Liu, C. Jia, and J. Williams, Event-triggered recursive state estimation for dynamical networks under randomly switching topologies and multiple missing measurements, Automatica, vol. 115 Article No: 108908, 2020. DOI: 10.1016/j.automatica.2020.108908.

[18] J. Hu, Z. Wang, G.-P. Liu, and H. Zhang, Variance-constrained recursive state estimation for time-varying complex networks with quantized measurements and uncertain inner coupling, IEEE Transactions on Neural Networks and Learning Systems, vol. 31, no. 6, pp. 1955-1967, 2020.

[19] J. Hu, Z. Wang, S. Liu, and H. Gao, A variance-constrained approach to recursive state estimation for time-varying complex networks with missing measurements, Automatica, vol. 64, pp. 155-162, 2016.

[20] C.-S. Hsieh and F.-C. Chen, Optimal solution of the two-stage Kalman estimator, IEEE Transactions on Automatic Control, vol. 44, no. 1, pp. 194-199, 1999.

[21] M. B. Ignagni, Separate-bias Kalman estimator with bias state noise, IEEE Transactions on Automatic Control, vol. 35, no. 3, pp. 338-341, 1990.

[22] M. B. Ignagni, Optimal and suboptimal separate-bias Kalman estimators for a stochastic bias, IEEE Transactions on Automatic Control, vol. 45, no. 3, pp. 547-551, 2000.

[23] K. H. Kim, J. G. Lee and C. G. Park, Adaptive two-stage Kalman filter in the presence of unknown random bias, International Journal of Adaptive Control and Signal Processing, vol. 20, no. 7, pp. 305-319, 2006.

[24] H. Lan, Y. Liang, F. Yang, Z. Wang and Q. Pan, Joint estimation and identification for stochastic systems with unknown inputs, IET Control Theory and Applications, vol. 7, no. 10, pp. 1377-1386, 2013.

[25] L. Li, H. Yang, Y. Xia, and H. Yang, State estimation for linear systems with unknown input and random false data injection attack, IET Control Theory and Applications, vol. 13, no. 6, pp. 823-831, 2019.

[26] J. Li, Z. Wang, H. Dong and G. Ghinea, Outlier-resistant remote state estimation for recurrent neural networks with mixed time-delays, IEEE Transactions on Neural Networks and Learning Systems, in press, DOI: 10.1109/TNNLS.2020.2991151.

[27] Q. Li, Z. Wang, W. Sheng, F. E. Alsaadi and F. E. Alsaadi, Dynamic event-triggered mechanism for $H_{\infty}$ non-Fragile state estimation of complex networks under randomly occurring sensor saturations, Information Sciences, vol. 509, pp. 304-316, Jan. 2020.

[28] W. Li, Y. Jia, and J. Du, Recursive state estimation for complex networks with random coupling strength, Neurocomputing, vol. 219, pp. 1-8, 2017.

[29] W. Li, Y. Jia, and J. Du, Variance-constrained state estimation for nonlinearly coupled complex networks, IEEE Transactions on Cybernetics, vol. 48, no. 2, pp. 818-824, 2018.

[30] G.-P. Liu, Consensus and stability analysis of networked multiagent predictive control systems, IEEE Transactions on Cybernetics, vol. 47, no. 4, pp. 1114-1119, 2017.

[31] M. Liu and H. Chen, $H_{\infty}$ state estimation for discrete-time delayed systems of the neural network type with multiple missing measurements, IEEE Transactions on Neural Networks and Learning Systems, vol. 26, no. 12, pp. 2987-2998, 2015.

[32] X. Liu, D. W. C. Ho, and C. Xie, Prespecified-time cluster synchronization of complex networks via a smooth control approach, IEEE Transactions on Cybernetics, vol. 50, no. 4, pp. 1771-1775, 2020.

[33] Y. Liu, Z. Wang, L. Ma and F. E. Alsaadi, A partial-nodes-based information fusion approach to state estimation for discrete-time delayed stochastic complex networks, Information Fusion, vol. 49, pp. 240-248, Sept. 2019.

[34] Y. Liu, Z. Wang, Y. Yuan and W. Liu, Event-triggered partial-nodesbased state estimation for delayed complex networks with bounded distributed delays, IEEE Transactions on Systems, Man, and CyberneticsSystems, vol. 49, no. 6, pp. 1088-1098, Jun. 2019.

[35] Y.-J. Liu, S.-C. Tong, C. L. P. Chen, and D-J. Li, Neural controller design-based adaptive control for nonlinear MIMO systems with unknown hysteresis inputs, IEEE Transactions on Cybernetics, vol. 46, no. 1, pp. 9-19, 2016.

[36] Z.-H. Pang, G.-P. Liu, D. Zhou, and D. Sun, Input design-based compensation control for networked nonlinear systems with random delays and packet dropouts, IEEE Transactions on Circuits and Systems II-Express Briefs, vol. 64, no. 3, pp. 299-303, 2017.

[37] M. Park, S.-H. Lee, O.-M. Kwon, and A. Seuret, Closeness-centralitybased synchronization criteria for complex dynamical networks with in- 
terval time-varying coupling delays, IEEE Transactions on Cybernetics, vol. 48, no. 7, pp. 2192-2202, 2018.

[38] T. E. Shirley, Treatment of bias in suboptimal filter analysis and recursive filtering, International Journal of Control, vol. 28, no. 3, pp. 457-465, 1978.

[39] B. Shen, Z. Wang, and H. Qiao, Event-triggered state estimation for discrete-time multidelayed neural networks with stochastic parameters and incomplete measurements, IEEE Transactions on Neural Networks and Learning Systems, vol. 28, no. 5, pp. 1152-1163, 2017.

[40] B. Shen, Z. Wang, D. Wang and Q. Li, State-saturated recursive filter design for stochastic time-varying nonlinear complex networks under deception attacks, IEEE Transactions on Neural Networks and Learning Systems, Vol. 31, No. 10, pp. 3788-3800, Oct. 2020.

[41] A. Vosughi, C. Johnson, M. Xue, S. Roy, and S. Warnick, Target control and source estimation metrics for dynamical networks, Automatica, vol. 100, pp. 412-416, 2019.

[42] F. Wang, Z. Wang, J. Liang, and X. Liu, Recursive state estimation for two-dimensional shift-varying systems with random parameter perturbation and dynamical bias, Automatica, vol. 112, Article No: 108658, 2020.

[43] L. Wang, Z. Wang, T. Huang, and G. Wei, An event-triggered approach to state estimation for a class of complex networks with mixed time delays and nonlinearities, IEEE Transactions on Cybernetics, vol. 46, no. 11 , pp. 2497-2508, 2016.

[44] S. Z. Yong, M. Zhu, and E. Frazzoli, A unified filter for simultaneous input and state estimation of linear discrete-time stochastic systems, Automatica, vol. 63, pp. 321-329, 2016.

[45] D. Zhao, Z. Wang, D. W. C. Ho and G. Wei, Observer-based PID security control for discrete time-delay systems under cyber-attacks, IEEE Transactions on Systems, Man, and Cybernetics-Systems, in press, DOI: $10.1109 /$ TSMC.2019.2952539.

[46] L. Zou, Z. Wang, J. Hu, and D. Zhou, Moving horizon estimation with unknown inputs under dynamic quantization effects, IEEE Transactions on Automatic Control, vol. 65, no. 12, pp. 5368-5375, 2020.

[47] L. Zou, Z. Wang, Q.-L. Han, and D.-H. Zhou, Moving horizon estimation for networked time-delay systems under Round-Robin protocol, IEEE Transactions on Automatic Control, vol. 64, no. 12, pp. 51915198, 2019.

[48] L. Zou, Z. Wang, Q.-L. Han, and D.-H. Zhou, Moving horizon estimation of networked nonlinear systems with random access protocol, IEEE Transactions on Systems, Man, and Cybernetics-Systems, 2019, DOI: 10.1109/TSMC.2019.2918002.

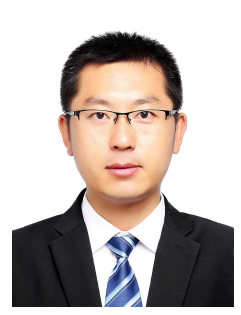

Jun Hu (M'14) received the B.Sc. degree in information and computation science and M.Sc. degree in applied mathematics from Harbin University of Science and Technology, Harbin, China, in 2006 and 2009, respectively, and the Ph.D. degree in control science and engineering from Harbin Institute of Technology, Harbin, China, in 2013.

From September 2010 to September 2012, he was a Visiting Ph.D. Student in the Department of Information Systems and Computing, Brunel University, U.K. From May 2014 to April 2016, he was an Alexander von Humboldt research fellow at the University of Kaiserslautern, Kaiserslautern, Germany. He is with the Department of Mathematics, Harbin University of Science and Technology, Harbin 150080, China, and also with the School of Engineering, University of South Wales, Pontypridd CF37 1DL, United Kingdom. His research interests include nonlinear control, filtering and fault estimation, time-varying systems and complex networks. He has published more than 60 papers in refereed international journals.

Dr. Hu serves as a reviewer for Mathematical Reviews, as an editor for Neurocomputing, Journal of Intelligent and Fuzzy Systems, Neural Processing Letters, Systems Science and Control Engineering, and as a guest editor for International Journal of General Systems and Information Fusion.

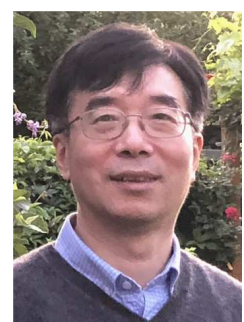

Zidong Wang (SM'03-F'14) was born in Jiangsu, China, in 1966. He received the B.Sc. degree in mathematics in 1986 from Suzhou University, Suzhou, China, and the M.Sc. degree in applied mathematics in 1990 and the Ph.D. degree in electrical engineering in 1994, both from Nanjing University of Science and Technology, Nanjing, China.

$\mathrm{He}$ is currently Professor of Dynamical Systems and Computing in the Department of Computer Science, Brunel University London, U.K. From 1990 to 2002, he held teaching and research appointments in universities in China, Germany and the UK. Prof. Wang's research interests include dynamical systems, signal processing, bioinformatics, control theory and applications. He has published 250+ papers in IEEE Transactions and $60+$ papers in Automatica. He is a holder of the Alexander von Humboldt Research Fellowship of Germany, the JSPS Research Fellowship of Japan, William Mong Visiting Research Fellowship of Hong Kong.

Prof. Wang serves (or has served) as the Editor-in-Chief for Neurocomputing, the Deputy Editor-in-Chief for International Journal of Systems Science, and an Associate Editor for 12 international journals including IEEE Transactions on Automatic Control, IEEE Transactions on Control Systems Technology, IEEE Transactions on Neural Networks, IEEE Transactions on Signal Processing, and IEEE Transactions on Systems, Man, and CyberneticsPart C. He is a Member of the Academia Europaea, a Fellow of the IEEE, a Fellow of the Royal Statistical Society and a member of program committee for many international conferences.

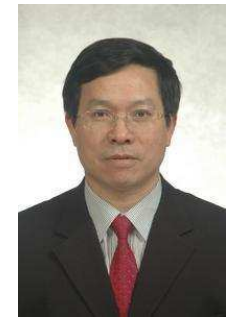

Guoping Liu (F'11) received the B.Eng and M.Eng degrees in automation from the Central South University, Changsha, China, in 1982 and 1985, respectively, and the Ph.D. degree in control engineering from the University of Manchester, Manchester, UK, in 1992. He is a professor with Wuhan University, China. His current research interests include networked multi-agent control systems, nonlinear system identification and control, advanced control of industrial systems, and multiobjective optimization and control. He has authored/co-authored over 200 journal papers and 10 books on control systems.

Prof. Liu is an editor-in-chief of the International Journal of Automation and Computing, IET Fellow and IEEE Fellow. 